\title{
LAW OF LARGE NUMBERS FOR A TWO-DIMENSIONAL CLASS COVER PROBLEM
}

\author{
Elvan Ceyhan ${ }^{1, *} \mathbb{D}$, John C. Wierman ${ }^{2}$ and Pengfei Xiang ${ }^{3}$
}

\begin{abstract}
We prove a Law of Large Numbers (LLN) for the domination number of class cover catch digraphs (CCCD) generated by random points in two (or higher) dimensions. DeVinney and Wierman (2002) proved the Strong Law of Large Numbers (SLLN) for the uniform distribution in one dimension, and Wierman and Xiang (2008) extended the SLLN to the case of general distributions in one dimension. In this article, using subadditive processes, we prove a SLLN result for the domination number generated by Poisson points in $\mathbb{R}^{2}$. From this we obtain a Weak Law of Large Numbers (WLLN) for the domination number generated by random points in $[0,1]^{2}$ from uniform distribution first, and then extend these result to the case of bounded continuous distributions. We also extend the results to higher dimensions. The domination number of CCCDs and related digraphs have applications in statistical pattern classification and spatial data analysis.
\end{abstract}

Mathematics Subject Classification. 60G99, 62H30, 05C69.

Received January 9, 2019. Accepted June 29, 2021.

\section{INTRODUCTION}

\subsection{The class cover problem}

The class cover problem (CCP) is motivated by its applications in pattern classification [18]. Its study was initiated by Cowen and Cannon in [3], and has been actively pursued, since its solution can directly be used to generate classifiers competitive with other methods in the literature (see [20, 23]).

We first give a formal description of the CCP: Let $\mathcal{X}_{n}=\left\{X_{1}, X_{2}, \ldots, X_{n}\right\}$ and $\mathcal{Y}_{m}=\left\{Y_{1}, Y_{2}, \ldots, Y_{m}\right\}$ be two sets of i.i.d. random variables taking values in a sample space $\Omega$ and are from classes $\mathcal{X}$ and $\mathcal{Y}$ with classconditional distribution functions $F_{X}$ and $F_{Y}$, respectively. Consider a dissimilarity function $\rho: \Omega \times \Omega \rightarrow \mathbb{R}_{+}$ such that $\rho(\alpha, \beta)=\rho(\beta, \alpha) \geq \rho(\alpha, \alpha)=0$ for all $\alpha, \beta \in \Omega$, where $\mathbb{R}_{+}$denotes the nonnegative real numbers. We assume $X_{i}$ 's are independent of $Y_{j}$ 's, and all $X_{i}$ 's and $Y_{j}$ 's are distinct with probability one. For each $X_{i}$, we define its covering ball $B\left(X_{i}\right)=\left\{\omega \in \Omega: \rho\left(X_{i}, \omega\right)<\min _{j} \rho\left(X_{i}, Y_{j}\right)\right\}$. A class cover of $\mathcal{X}_{n}$ is a subset of covering balls whose union contains all $X_{i}$ 's. Obviously, the set consisting of all covering balls of $X_{i}$ 's constitutes a class

Keywords and phrases: Pattern classification, class cover catch digraph, domination number, law of large numbers, subadditive process.

${ }^{1}$ Department of Mathematics and Statistics, Auburn University, Auburn, AL 36849, USA.

2 Department of Applied Mathematics and Statistics, The Johns Hopkins University, Baltimore, MD 21218, USA.

3 China Securities, 188 Chao Nei St., Dong Cheng District, Beijing 10010, P.R. China.

* Corresponding author: ceyhan@auburn.edu 
cover for class $\mathcal{X}$. However, we want to find a cover of class $\mathcal{X}$ that is as small as possible. Therefore, the CCP we consider is equivalent to finding a minimum cardinality class cover. DeVinney [8] proved that solving the $\mathrm{CCP}$ is in general an NP-hard problem.

Furthermore, the CCP can be converted to a purely graph theoretic problem of finding minimum dominating sets. In a digraph $D=(V, A)$, a vertex $u \in V$ dominates itself and all vertices of the form $\{v:(u, v) \in A\}$. A dominating set $S_{D}$ for the digraph $D$ is a subset of $V$ such that each vertex $u \in V$ is dominated by a vertex in $S_{D}$. A minimum dominating set $S_{D}^{*}$ is a dominating set of minimum cardinality and the domination number $\gamma(D)$ is defined as $\gamma(D):=\left|S_{D}^{*}\right|$, where $|\cdot|$ denotes the set cardinality functional. The class cover catch digraph $(C C C D)$ induced by a CCP for a realization of $\mathcal{X}_{n}$ is the digraph $D=(V, A)$ with the vertex set $V=\mathcal{X}_{n}$ and there is an $\operatorname{arc}\left(x_{i}, x_{j}\right)$ in arc set $A$ if and only if $x_{j} \in B\left(x_{i}\right)$. It is easy to see that the CCP is equivalent to "finding a minimum dominating set of the induced CCCD and using the points in the minimum dominating set to construct the minimum cover". More discussion on graph domination is provided in [15].

The solutions of the CCP can be used to build classifiers. For example, the balls around the members of the minimum dominating sets of the CCCDs can be used to construct discriminant regions for assigning class labels (see [8] for more detail). In this setting, we want to choose a class cover to represent class $\mathcal{X}$ that is as small as possible (i.e., a minimum dominating set for $\mathcal{X}_{n}$ ), to make the classifier less complex while keeping most of the relevant information. The use of the minimum dominating sets of the CCCDs in classification are illustrated in $[9,20,23]$. CCCDs can also be used to determine the underlying or inherent scale dimension for high dimensional data [19]. Eveland et al. proposed a boosted version of CCCD classifiers which are efficient and fast in computation as well [12]. CCCD classifiers are also used in DNA microarray analysis in [24]. CCCDs are generalized to random geometric digraphs called proximity catch digraphs (PCDs) in [4] and the minimum dominating sets of PCDs are also proposed as solutions to the CCP problem, and the cardinality of the minimum dominating sets (i.e., domination number) of the PCDs is used as a test statistic for testing spatial clustering in a multi-class setting $[4,6]$. The arc density (i.e., number of arcs in a digraph divided by the total number of arcs possible in a digraph of the same order) of PCDs is also used for the same purpose (see [7] and references therein).

The mild conditions for the dissimilarity function $\rho$ are sufficient to define the CCP, CCCDs, and the dominating sets and domination number of CCCDs. However, throughout the rest of the article, we take $\rho$ to be the regular Euclidean distance, although most of the arguments would work if it were only taken to be an increasing function of the Euclidean distance. The type of the balls depend on the distance or dissimilarity function chosen, but given the dissimilarity measure, if the change in the radius of the balls do not alter the induced CCCD, then the solution(s) to the CCP problem would be the same points and balls with the same centers.

Recently a random geometric graph called secrecy graph was also introduced to model a wireless network for secure communication (see $[13,27]$ ). Taking class $\mathcal{X}$ points as the "good guys" (i.e., communicators over the networks), and class $\mathcal{Y}$ points as "bad guys" (i.e., eavesdroppers in the network), an arc is inserted between two $\mathcal{X}$ subjects $x_{i}$ and $x_{j}$ if there are no eavesdroppers falling in the radius of the ball centered at $x_{i}$ with radius being the distance between $x_{i}$ and $x_{j}$. A quick investigation shows that the directed secrecy graph is equivalent to the CCCD; the basic secrecy graph is the reflexivity graph (in which edges are inserted when two vertices have two distinct arcs between them); and the enhanced secrecy graph is equivalent to the usual underlying graph of the CCCD. The authors in $[13,27]$ study the percolation of the connectivity of these secrecy graphs under the lattice and Poisson models for the vertices.

\subsection{Previous results}

The domination number of a CCCD is the cardinality of a minimum dominating set of the CCCD. In 1962, Ore [21] first used the term "domination number". Due to the many applications of the domination number in fields such as computer networks, social sciences and computational complexity, there has been increasing interest on this topic (see, e.g., $[2,26,34])$. In the CCCD setting, we denote the domination number by $\Gamma\left(\mathcal{X}_{n}, \mathcal{Y}_{m}\right)$ to indicate dependence on $\mathcal{X}_{n}$ and $\mathcal{Y}_{m}$. 
DeVinney and Wierman [10] proved the following SLLN for the special case of $\Omega=\mathbb{R}, F_{X}=F_{Y}=U[0,1]$, the uniform distribution on $[0,1]$ and in this special case the domination number is denoted by $\Gamma_{n, m}$ for brevity in notation.

Theorem 1.1. If $\Omega=\mathbb{R}, F_{X}=F_{Y}=U[0,1]$, and $m=\lfloor$ rn $\rfloor$, where $r \in(0, \infty)$, then

$$
\lim _{n \rightarrow \infty} \frac{\Gamma_{n, m}}{n}=g_{1}(r)=\frac{r(12 r+13)}{3(r+1)(4 r+3)} \text { a.s. }
$$

Wierman and Xiang [31] extended this result to the case of general distributions in one dimension, as stated in the following theorem. In this case, $f_{X}$ and $f_{Y}$ are probability density functions (pdfs) corresponding to $\mathcal{X}_{n}$ and $\mathcal{Y}_{m}$, respectively.

Theorem 1.2. If $\Omega=\mathbb{R}$, the continuous and bounded pdfs $f_{X}$ and $f_{Y}$ have support $[a, b]$ with $a<b$, and $m / n \rightarrow r, r \in(0, \infty)$ as $n \rightarrow \infty$, then

$$
\lim _{n \rightarrow \infty} \frac{\Gamma\left(\mathcal{X}_{n}, \mathcal{Y}_{m}\right)}{n}=\int_{a}^{b} g_{1}\left(r \cdot \frac{f_{Y}(u)}{f_{X}(u)}\right) \cdot f_{X}(u) \mathrm{d} u \text { a.s. }
$$

where $g_{1}(r)$ is defined as in Theorem 1.1.

\subsection{Our results}

Extending the previous results in one dimension to higher dimensions requires a different approach, since the exact distribution of the domination number in the multi-dimensional case is unknown. In this paper, we first focus on $\mathbb{R}^{2}$ and develop the LLN for the domination number in $\mathbb{R}^{2}$ by using the ergodic theorem for twodimensional subadditive processes. Then we extend the results to higher dimensions in Section 6. See [25, 30] for the subadditive Euclidean functionals. Our approach is to prove the SLLN for the domination number of the CCCD generated by the Poisson points in $\mathbb{R}^{2}$, and then transfer the result to uniform data sets on $[0,1]^{2}$ with fixed sample sizes. Our arguments are similar to those given in $[22,25,30,33]$. In particular, the subadditive approximation is also known as the boundary functional. Furthermore, the de-Poissonization argument we employ was introduced in [33]. However, the domination number in the Poisson case is not subadditive. To make use of the SLLN for subadditive processes, we construct the constrained domination number of the CCCD induced by the $X$-points and $Y$-points with the covering balls bounded also by the boundary of the study region, which is assumed to be a rectangular region $R$ in $\mathbb{R}^{2}$. Below, we define the domination number and constrained domination number based on CCCDs generated by realizations of $\mathcal{X}_{n}$ and $\mathcal{Y}_{m}$ in region $R$. To emphasize the distinction between the two versions, the former will also be called unconstrained domination number when there is potential ambiguity.

Definition 1.3. (Unconstrained) Domination Number: For a rectangle $R$ in $\mathbb{R}^{2}$, the (unconstrained) covering ball of any $x_{i} \in R$ is defined by

$$
B_{R}\left(x_{i}\right)=\left\{\omega \in \Omega: \rho\left(x_{i}, \omega\right)<\min _{y_{j} \in R} \rho\left(x_{i}, y_{j}\right)\right\} .
$$

The (unconstrained) domination number $\Gamma_{R}$ is the minimum number of (unconstrained) covering balls needed to cover all $X$-points in $R \subseteq \mathbb{R}^{2}$.

Definition 1.4. Constrained Domination Number: For a rectangle $R$ with boundary $\partial(R)$, the constrained covering ball of any $x_{i} \in R$ is defined by

$$
\bar{B}_{R}\left(x_{i}\right)=\left\{\omega \in \Omega: \rho\left(x_{i}, \omega\right)<\min \left\{\min _{y_{j} \in R} \rho\left(x_{i}, y_{j}\right), \min _{z \in \partial(R)} \rho\left(x_{i}, z\right)\right\}\right\} .
$$


The constrained domination number $\bar{\Gamma}_{R}$ is the minimum number of constrained covering balls needed to cover all $X$-points in $R$.

Let $\mathbf{S}^{2}=\mathbb{R}_{+}^{2}$ be the two-dimensional quadrant with nonnegative real coordinates. If $\mathbf{a}=\left(a_{i}\right)$ and $\mathbf{b}=\left(b_{i}\right)$ for $i=1,2$ are two vectors in $\mathbf{S}^{2}$ then $[\mathbf{a}, \mathbf{b})$ denotes the set $\left\{\mathbf{u}: \mathbf{u}=\left(u_{i}\right) \in \mathbf{S}^{2}, a_{i} \leq u_{i}<b_{i}, i=1,2\right\}(i . e .,[\mathbf{a}, \mathbf{b})$ denotes a rectangle $R$ which is assumed to include the left edge but exclude the right edge in each coordinate) and let $\mathfrak{R}$ denote the class of sets of this form. Denote $\mathbf{0}$ (and $\mathbf{e}$ ) as the vectors with all coordinates equal to 0 (and 1). Let $J_{q}=\left[\mathbf{0}, q \mathbf{e}\right.$ ), for $q>0$, and $\left|J_{q}\right|$ denote its area. Notice that (the closure of) $J_{q}$ is a square with lower left vertex at origin 0 and side length $q$, so its area is $\left|J_{q}\right|=q^{2}$. Suppose that there are two independent Poisson processes $\left\{X_{i}\right\}$ and $\left\{Y_{j}\right\}$ in $\mathbb{R}^{2}$, with rates $\lambda_{X}$ and $\lambda_{Y}$, respectively. Notice that the number of $X$-points on $J_{q}$, denoted $N_{X}\left(J_{q}\right)$ has a Poisson distribution, Poisson $\left(\lambda_{X}\left|J_{q}\right|\right) \equiv \operatorname{Poisson}\left(q^{2} \lambda_{X}\right)$. Similarly, the number of $Y$-points on $J_{q}$, denoted $N_{Y}\left(J_{q}\right)$ has Poisson $\left(q^{2} \lambda_{Y}\right)$ distribution. Throughout the article, we use $\lim _{q \rightarrow \infty, q \in A}$ for the limit as $q$ tending to infinity through the elements of $A$ (e.g., if $A=\mathbb{Q}$ means the limit is "as $q$ tends to infinity through rational numbers"), and $\lim _{q \rightarrow \infty}$ with no specification for $q$ for the limit as $q$ tending to infinity through the integers. In Section 2, by directly applying the SLLN for subadditive processes and the separability argument, we establish the following SLLN result for the constrained domination number in the Poisson case.

Theorem 1.5. Let $\left\{X_{i}\right\}$ and $\left\{Y_{j}\right\}$ be two independent Poisson processes in $\mathbb{R}^{2}$, with rates $\lambda_{X}$ and $\lambda_{Y}$, respectively. Then if $\lambda_{Y} / \lambda_{X}=r$ for $r \in(0, \infty)$, there exists a function $g_{2}(r)$ such that $\lim _{t \rightarrow \infty, t \in \mathbb{R}} \frac{\bar{\Gamma} J_{t}}{\left|J_{t}\right|}=g_{2}(r)$ a.s.

Although the explicit form of $g_{2}(r)$ is not available, in Corollary 4.2 we show that the function $g_{2}(r)$ is bounded, increasing and continuous in $(0, \infty)$. Furthermore, the conclusion of Theorem 1.5 also holds for the (unconstrained) domination number with the square study region with real vertices:

Theorem 1.6. Let $\left\{X_{i}\right\}$ and $\left\{Y_{j}\right\}$ be two independent Poisson processes in $\mathbb{R}^{2}$, with rates $\lambda_{X}$ and $\lambda_{Y}$, respectively. Then if $\lambda_{Y} / \lambda_{X}=r$ for $r \in(0, \infty)$, then $\lim _{t \rightarrow \infty, t \in \mathbb{R}} \frac{\Gamma_{J_{t}}}{\left|J_{t}\right|}=g_{2}(r)$ a.s., where $g_{2}(r)$ is as in Theorem 1.5 .

Denote $\Gamma_{n, m}$ as the domination number generated by $n X$-points and $m Y$-points where both $X$-points and $Y$-points are uniformly distributed in the unit square, denoted $U[0,1]^{2}$. In Section 4 , by viewing the Poisson points as uniformly distributed points in $[0,1]^{2}$ (that is, coupling the Poisson point process with a binomial point process and, in particular, approximating the Poisson distribution by a binomial distribution), we prove the following Weak Law of Large Numbers (WLLN) for the domination number in $[0,1]^{2}$.

Theorem 1.7. Let $X_{i} \stackrel{i i d}{\sim} U[0,1]^{2}$ and $Y_{j} \stackrel{i i d}{\sim} U[0,1]^{2}$ and $X_{i}$ and $Y_{j}$ be independent for all $i=1,2, \ldots, n$ and $j=1,2, \ldots, m$. If $m / n \rightarrow r, r \in(0, \infty)$, as $n \rightarrow \infty$, then $\lim _{n \rightarrow \infty} \frac{\Gamma_{n, m}}{n}=g_{2}(r)$ in probability, where $g_{2}(r)$ is as in Theorem 1.5 .

Finally in Section 5, based on the approach used in the one dimensional version of this problem in [31], we generalize the WLLN to the case where the strictly positive and bounded densities $f_{X}$ and $f_{Y}$ with support $[0,1]^{2}$. Define

$$
\mathcal{L}_{2}\left(r, f_{1}, f_{2}\right):=\iint_{[0,1]^{2}} g_{2}\left(r \cdot \frac{f_{2}(u, v)}{f_{1}(u, v)}\right) \cdot f_{1}(u, v) \mathrm{d} u \mathrm{~d} v
$$

where $g_{2}(r)$ is as in Theorem 1.5 .

Theorem 1.8. Let $X_{i} \stackrel{\text { iid }}{\sim} f_{X}$ and $Y_{j} \stackrel{i i d}{\sim} f_{Y}$ and $X_{i}$ and $Y_{j}$ be independent for all $i=1,2, \ldots, n$ and $j=$ $1,2, \ldots, m$. If the pdfs $f_{X}$ and $f_{Y}$ are strictly positive, bounded and continuous on their support, [0,1 $]^{2}$, and 
$m / n \rightarrow r, r \in(0, \infty)$, as $n \rightarrow \infty$, then

$$
\lim _{n \rightarrow \infty} \frac{\Gamma_{n, m}}{n}=\mathcal{L}_{2}\left(r, f_{X}, f_{Y}\right) \text { in probability. }
$$

The dependent variables (i.e., constrained and unconstrained domination numbers) in this paper are negatively associated. See [32] for more details on negatively associated random variables and vectors. Therefore, it is possible to prove CLT results for these two domination numbers. However, currently we only know some properties of $g_{2}(r)$ (see Cor. 4.2.), hence, the asymptotic distribution (more specifically, CLT) is not pursued in this article.

\section{SLLN for the Domination number in the Poisson CASE}

Our proof relies on the ergodic theory of multidimensional subadditive stochastic processes. Subadditive processes were introduced by Hammersley and Welsh in [14] and developed by Kingman in [16, 17]. In 1981, Akcoglu and Krengel obtained a SLLN result for multidimensional subadditive processes under several natural assumptions [1], which we employ in our proof. We state their results in terms of subadditive, instead of superadditive processes below:

Definition 2.1. Let $\mathfrak{T}$ denote the class of sets of the form $[\mathbf{a}, \mathbf{b}):=\left\{\mathbf{u}: \mathbf{u}=\left(u_{i}\right) \in \mathbf{S}^{d}, a_{i} \leq u_{i}<b_{i}\right\}$ for two vectors $\mathbf{a}=\left(a_{i}\right)$ and $\mathbf{b}=\left(b_{i}\right)$ in $\mathbf{S}^{d}=\mathbb{R}_{+}^{d}$, the additive semigroup of $d$-dimensional vectors with nonnegative real coordinates where $d \in \mathbb{N}$. A continuous subadditive process $\left\{X_{I}: I \in \mathfrak{T}\right\}$ satisfies:

S1: If $I_{1}, I_{2}, \ldots, I_{k}$ are disjoint sets in $\mathfrak{T}$, and $I=\cup_{i=1}^{k} I_{i}$ is also in $\mathfrak{T}$, then $X_{I} \leq \sum_{i=1}^{k} X_{I_{i}}$.

S2: For $I_{1}, I_{2}, \ldots, I_{k} \in \mathfrak{T}$, and $\mathbf{u} \in \mathbf{S}^{d}$, the joint distributions of $\left(X_{I_{1}}, \ldots, X_{I_{k}}\right)$ and $\left(X_{\mathbf{u}+I_{1}}, \ldots, X_{\mathbf{u}+I_{k}}\right)$ are identical where $\mathbf{u}+I_{j}=\left\{\mathbf{u}+\mathbf{a}: \mathbf{a} \in I_{j}\right\}$ for $j=1,2, \ldots, k$.

S3: $\mathbf{E}\left[X_{I}\right]<\infty$ for all $I \in \mathfrak{T}$ and $\inf \left\{\mathbf{E}\left[X_{I}\right] /|I|: I \in \mathfrak{T},|I|>0\right\}=\tau(X)>-\infty$,

where $|\cdot|$ denotes the Lebesgue measure and $\tau(X)$ is referred to as the time constant of the stochastic process $\left\{X_{I}\right\}$.

Let $\mathbf{S}_{\mathbb{Z}}^{d}$ denote the set of vectors in $\mathbf{S}^{d}$ with integer coordinates. For a real number $t>0$, let $\mathbf{S}_{t}^{d}:=\left\{t \mathbf{u}: \mathbf{u} \in \mathbf{S}_{\mathbb{Z}}^{d}\right\}$ and $\mathfrak{T}_{t}:=\left\{[\mathbf{a}, \mathbf{b}): \mathbf{a}, \mathbf{b} \in \mathbf{S}_{t}^{d}\right\}$. If $\left\{X_{I}\right\}$ is defined only on $\mathfrak{T}_{t}$ for some fixed $t>0$, and satisfies conditions $\mathbf{S 1 - S 3}$, then it is called a discrete subadditive process. Akcoglu and Krengel [1] proved the following theorem for the $t=1$ case:

Theorem 2.2. If $\left\{X_{I}\right\}$ is a discrete subadditive process on $\mathfrak{T}_{1}$, then $\lim _{n \rightarrow \infty} \frac{X_{J_{n}}}{\left|J_{n}\right|}$ exists a.e., where $J_{n}=[\mathbf{0}, n \mathbf{e}) \in$ $\mathfrak{T}_{1}$.

Kingman observed that the continuous analogue of Theorem 2.2 is false unless further conditions are added and proposed a natural supplementary condition [16, 17]. The following theorem gives a multi-parameter generalization of the result in [1].

Theorem 2.3. Suppose $\left\{X_{I}\right\}$ is a subadditive process on rectangles with rational end points. Let $J_{s}=[\mathbf{0}$, se) for $s>0$ and $\Phi=\sup \left|X_{I}\right|$ where the supremum is taken over all rectangles with rational end points in $J_{1}=[\mathbf{0}, \mathbf{e})$. If $\mathbf{E}[\Phi]<\infty$, then $\lim _{q \rightarrow \infty, q \in \mathbb{Q}} \frac{X_{J_{q}}}{\left|J_{q}\right|}$ exists a.e.

To eliminate the restriction of $q$ to rational numbers, we rely on the concept of separability. A stochastic process $\left\{Y_{t}, t \in T\right\}$ is separable if the parameter set $T$ has a countable dense subset $D$ and there is an event $E$ with probability zero, so that for every open set $F \subset T$ and every closed set $G \subset \mathbb{R}$, the events $\left\{Y_{t} \in G, \forall t \in F \cap D\right\}$ and $\left\{Y_{t} \in G, \forall t \in F\right\}$ differ by a subset of $E$. Doob [11] introduced separability to describe the condition that the properties of a stochastic process are determined by its values at a countable set of parameter values. Since $\left\{X_{I_{q}}\right\}$ is constant except for jumps at the Poisson points, it is clearly a separable process. Hence, with $D$ taken 
to be the rational numbers, a.s. convergence of $\frac{X_{J_{q}}}{\left|J_{q}\right|}$ as $q$ tends to infinity through the rational numbers implies a.s. convergence through the real numbers.

Theorem 2.2 alone does not identify the limiting random variable $\mathcal{L}_{d}:=\lim _{|I| \rightarrow \infty} \frac{X_{I}}{|I|}$ for a subadditive process $\left\{X_{I}\right\}$. However, the limit is simply the time constant $\tau(X)$ when the subadditive process is independent, where a subadditive process is defined to be independent if the random variables $\left\{X_{I_{i}}\right\}$ are independent for disjoint regions $\left\{I_{i}\right\}$ for $i=1,2, \ldots, k$ (see $[16,17]$ ).

Consider the stochastic process $\left\{\bar{\Gamma}_{R}: R=[\mathbf{a}, \mathbf{b}), \mathbf{a}, \mathbf{b}\right.$ have nonnegative rational components $\}$ where $\bar{\Gamma}_{R}$ is the cardinality of the class cover of the $X$-points with constrained covering balls in $R$. We prove that

Lemma 2.4. $\left\{\bar{\Gamma}_{R}\right\}$ is a subadditive process.

Proof. In this setting, $\mathfrak{T}$ in Definition 2.1 is the set of regions $[\mathbf{a}, \mathbf{b})$ with $\mathbf{a}$ and $\mathbf{b}$ having nonnegative rational components. The set of such regions is denoted by $\mathfrak{R}_{q}$. We check the three conditions, $\mathbf{S} \mathbf{1}-\mathbf{S} 3$, in Definition 2.1 for $\left\{\bar{\Gamma}_{R}: R \in \mathfrak{R}_{q}\right\}$ as follows:

- For $\mathbf{S 1}$, suppose that $R_{1}, R_{2}, \ldots, R_{k}$ are disjoint regions in $\mathfrak{R}_{q}$ and that $R=\cup_{i=1}^{k} R_{i}$ is in $\mathfrak{R}_{q}$ as well. If a point $X \in R$, then there exists a $j \in\{1,2, \ldots, n\}$ such that $X \in R_{j}$. The constrained covering ball for $X$ with respect to $R$, denoted $\bar{B}_{R}(X)$, is the same as or larger than that with respect to $R_{j}$, denoted $\bar{B}_{R_{j}}(X)$. Hence, no constrained covering ball with respect to $R$ is any smaller than its corresponding constrained covering ball with respect to $R_{j}$. Thus, the minimum number of constrained covering balls required to cover all $X$-points in $R$ would not be larger than that required to cover all $X$-points in $R_{j}$ 's. Therefore, after ignoring the boundaries of $R_{j}$ 's, the union of the new constrained covering balls (with respect to $R$ ) still contains all $X$-points in $R_{j}$ 's, and thus all $X$-points in $R$. Hence, it follows that, $\bar{\Gamma}_{R} \leq \sum_{i=1}^{n} \bar{\Gamma}_{R_{i}}$.

- S2 follows from the homogeneity of Poisson processes.

- S3 holds, since $\mathbf{E}\left[\bar{\Gamma}_{R}\right]>0$ for any $R$ and $\bar{\Gamma}_{R}$ is bounded.

Next, we prove Theorem 1.5 by applying Theorem 2.3 to the process $\left\{\bar{\Gamma}_{J_{q}}\right\}$ where $J_{q}=[\mathbf{0}, q \mathbf{e})$ where $q$ is rational and then extending this result to $J_{t}$ where $t$ is real.

Proof of Theorem 1.5. We first consider $\bar{\Gamma}_{J_{q}}$ with $q$ being rational. To apply Theorem 2.3 , we just need to check that $\mathbf{E}[\Phi]<\infty$, where $\Phi=\sup \left|\bar{\Gamma}_{R}\right|$ with the supremum taken over all rectangles $R$ with rational end points in $J_{1}$. For any rational number $q \leq 1$, we have $\bar{\Gamma}_{J_{q}} \leq N_{X}\left(J_{q}\right) \leq N_{X}\left(J_{1}\right)$. Thus $\Phi \leq N_{X}\left(J_{1}\right)$. Taking the expectation of both sides, we have $\mathbf{E}[\Phi] \leq \mathbf{E}\left[N_{X}\left(J_{1}\right)\right]=\lambda_{X}\left|J_{1}\right|=\lambda_{X}<\infty$. Hence, by Theorem 2.3,

$$
\lim _{q \rightarrow \infty, q \in \mathbb{Q}} \frac{\bar{\Gamma}_{J_{q}}}{\left|J_{q}\right|} \text { exists a.s. }
$$

Furthermore, the subadditive process $\left\{\bar{\Gamma}_{J_{q}}: q\right.$ rational $\}$ is independent because $\bar{\Gamma}_{R_{i}}$ and $\bar{\Gamma}_{R_{j}}$ are independent for disjoint rectangles $R_{i}$ and $R_{j}$. Thus

$$
\lim _{q \rightarrow \infty, q \in \mathbb{Q}} \frac{\bar{\Gamma}_{J_{q}}}{\left|J_{q}\right|}=\tau(\bar{\Gamma}) \text { a.s. }
$$

Notice that the time constant $\tau(\bar{\Gamma})$ of the stochastic process $\left\{\bar{\Gamma}_{J_{q}}\right\}$ depends on $r$ and $J_{q} \subset \mathbb{R}^{2}$, so we denote the time constant as $g_{2}(r)$. Since the process $\left\{\bar{\Gamma}_{R}\right\}$ is separable, a.s. convergence of $\frac{\bar{\Gamma}_{J_{q}}}{\left|J_{q}\right|}$ along each sequence of rational numbers implies a.s. convergence of $\frac{\bar{\Gamma}_{J_{t}}}{\left|J_{t}\right|}$ for all positive real numbers, since rational numbers form a dense countable set in the real numbers. Hence, the desired result follows. 


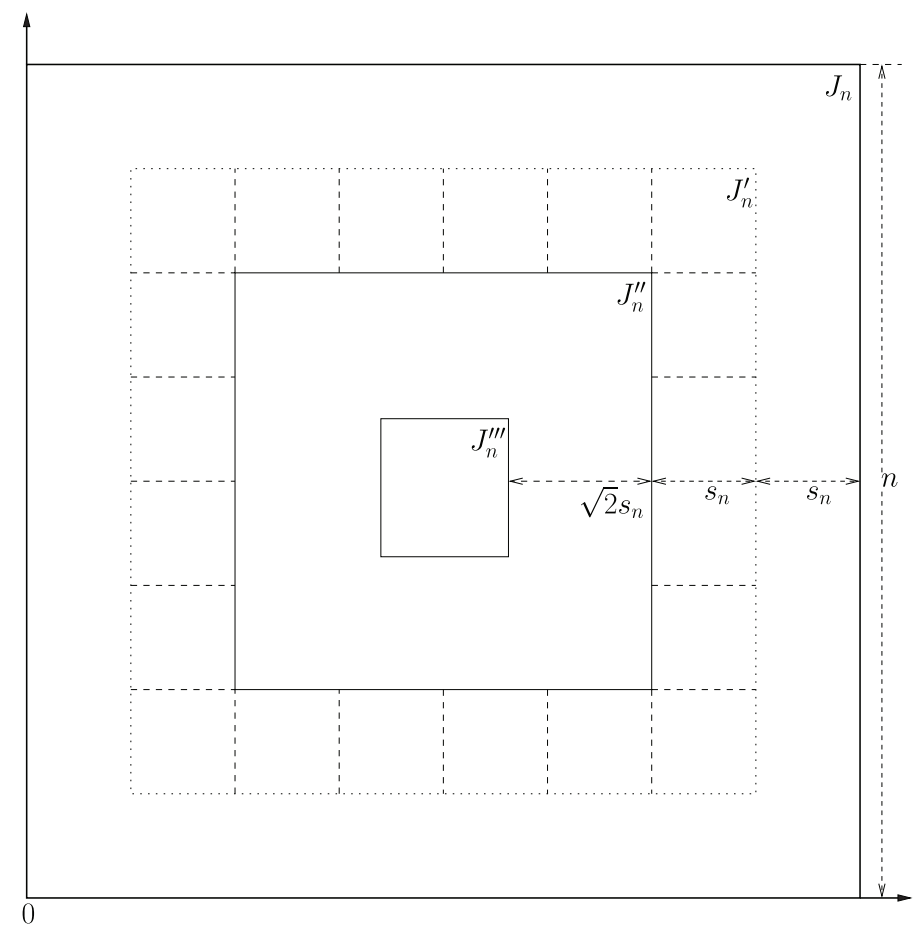

Figure 1. An illustration of the regions $J_{n}, J_{n}^{\prime}, J_{n}^{\prime \prime}$, and $J_{n}^{\prime \prime \prime}$.

\section{Proof of Theorem 1.6}

In Theorem 1.6, as $\lambda_{Y} / \lambda_{X}=r$, we have $\lim _{t \rightarrow \infty, t \in \mathbb{R}} \frac{\Gamma_{t}}{\left|J_{t}\right|}=g_{2}(r)$ a.s.

Having established the convergence result for the constrained domination number generated by the Poisson points, we are now ready to prove a similar result for the (unconstrained) domination number, i.e. prove Theorem 1.6. First, we prove a lemma that shows that the constrained and unconstrained domination numbers agree in the limit over $J[\mathbf{0}, n \mathbf{e})$ as $n \rightarrow \infty$.

Lemma 3.1. Let $J_{n}:=[\mathbf{0}, n \mathbf{e})$ where $n$ is a positive integer and denote the constrained domination number by $\bar{\Gamma}_{J_{n}}$ and the unconstrained domination number as $\Gamma_{J_{n}}$. Then $\lim _{n \rightarrow \infty} \frac{\Gamma_{J_{n}}}{\left|J_{n}\right|}=\lim _{n \rightarrow \infty} \frac{\bar{\Gamma}_{J_{n}}}{\left|J_{n}\right|}$ a.s.

Proof. Let $n$ be a positive integer and $s_{n}$ be a positive real number depending on $n$. Consider $J_{n}=[\mathbf{0}, n \mathbf{e})$, $J_{n}^{\prime}=\left[s_{n} \mathbf{e},\left(n-s_{n}\right) \mathbf{e}\right), J_{n}^{\prime \prime}=\left[2 s_{n} \mathbf{e},\left(n-2 s_{n}\right) \mathbf{e}\right)$, and $J_{n}^{\prime \prime \prime}=\left[(2+\sqrt{2}) s_{n} \mathbf{e},\left(n-(2+\sqrt{2}) s_{n}\right) \mathbf{e}\right)$ as shown in Figure 1 . The quantity $s_{n}<\frac{n}{2(2+\sqrt{2})}$ will be chosen later in the proof and we will let it go to infinity together with $n$ as $n \rightarrow \infty$ but at a much slower rate. Let $F_{n}$ denote the event that all constrained covering balls $\bar{B}_{J_{n}}\left(X_{i}\right)$ of $X$-points in $J_{n}^{\prime \prime}$ are contained in $J_{n}$, and let $E_{n}$ denote the event that there exists at least one $Y$-point in each of the $s_{n} \times s_{n}$ squares between $J_{n}^{\prime}$ and $J_{n}^{\prime \prime}$.

The probability of having at least one $Y$-point in one of those small squares is $1-\exp \left(-s_{n}^{2} \lambda_{Y}\right)$, and the number of small squares is $\left\lfloor 4 n / s_{n}-12\right\rfloor$ which is less than $4 n / s_{n}$. Therefore, by the independent increments property of Poisson processes, we know that

$$
P\left(E_{n}\right) \geq\left(1-\exp \left(-s_{n}^{2} \lambda_{Y}\right)\right)^{4 n / s_{n}}
$$




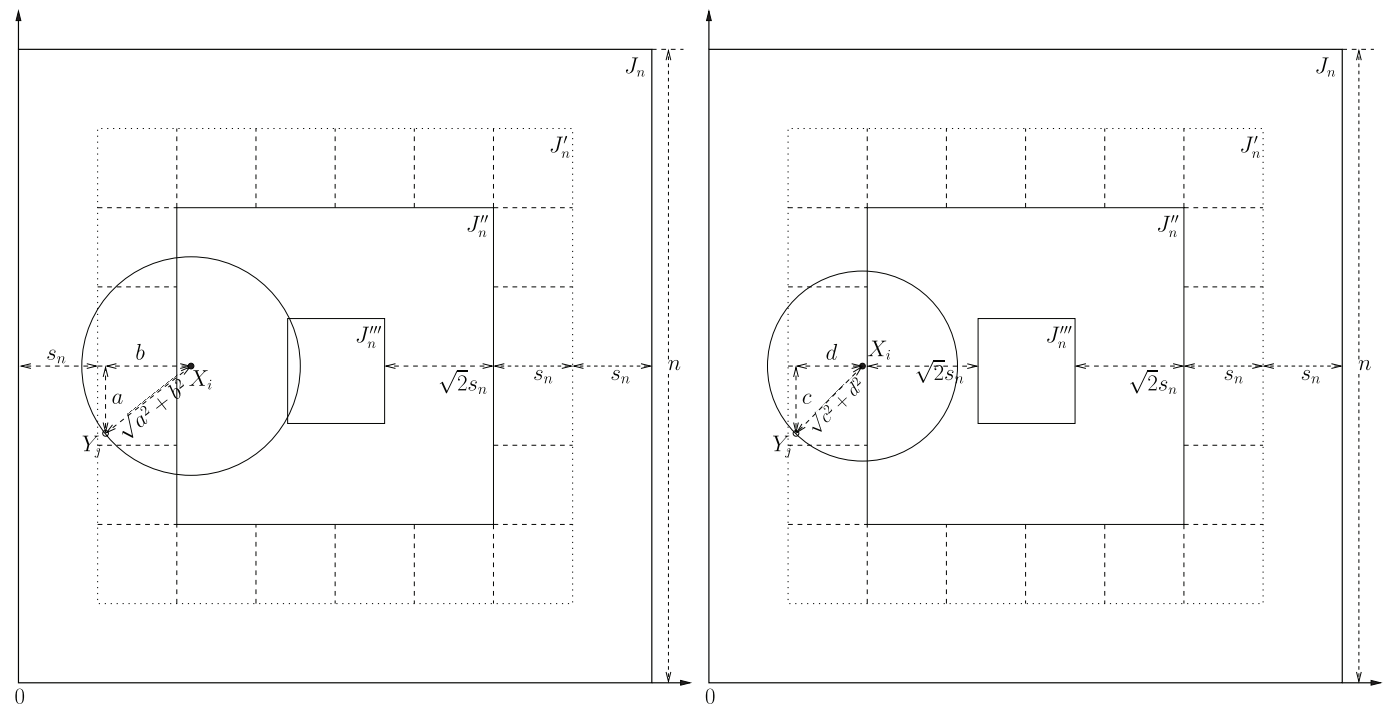

FIgURE 2. An illustration of $E_{n} \subseteq F_{n}$ (left) and an illustration of $\Delta_{J_{n}} \leq N_{X}\left(J_{n} \backslash J_{n}^{\prime \prime \prime}\right)($ right).

Next, we show that $E_{n} \subseteq F_{n}$, which will imply

$$
P\left(F_{n}\right) \geq\left(1-\exp \left(-s_{n}^{2} \lambda_{Y}\right)\right)^{4 n / s_{n}} .
$$

If there is at least one $Y$-point in each $s_{n} \times s_{n}$ square between $J_{n}^{\prime}$ and $J_{n}^{\prime \prime}$, then the constrained covering ball of any $X$-point in $J_{n}^{\prime \prime}$ cannot cross the boundary of $J_{n}$ (i.e., the constrained covering ball would stay in $\left.J_{n}\right)$. The reason is that for any $X_{i} \in J_{n}^{\prime \prime}$, the constrained covering ball $\bar{B}_{J_{n}}\left(X_{i}\right)$ cannot get very far away from $J_{n}^{\prime}$, since there is at least one point $Y_{j}$ in the closest $s_{n} \times s_{n}$ square to $X_{i}$. Hence, $\bar{B}_{J_{n}}\left(X_{i}\right)$ is contained in $J_{n}$. Specifically (but without loss of generality), suppose $Y_{j}$ is the $Y$-point closest to $X_{i}$, located at the position shown in Figure 2 (left). Then the radius of the constrained covering ball $\bar{B}_{J_{n}}\left(X_{i}\right)$ is $\sqrt{a^{2}+b^{2}}$, where the two segments with respective lengths $a$ and $b$ are also shown in Figure 2 (left). Since $a \leq s_{n}$, we have $\sqrt{a^{2}+b^{2}} \leq \sqrt{s_{n}^{2}+b^{2}} \leq b+s_{n}$. Note that the distance from $X_{i}$ to the the boundary of $J_{n}$ is greater or equal than $b+s_{n}$, thus $\bar{B}_{J_{n}}\left(X_{i}\right)$ is contained in $J_{n}$.

Next we carefully analyze the relation between the constrained domination number $\bar{\Gamma}_{J_{n}}$ and the (unconstrained) domination number $\Gamma_{J_{n}}$. Let $\Delta_{J_{n}}=\bar{\Gamma}_{J_{n}}-\Gamma_{J_{n}}$. If the boundary constraint is ignored, the constrained covering balls will not decrease (and might increase) for those $X$-points whose constrained covering balls touch the boundary; thus, the domination number will not increase, i.e. $\Delta_{J_{n}} \geq 0$. Hence, given the event $F_{n}$, the constrained covering ball resizing can only occur for those $X$-points in $J_{n} \backslash J_{n}^{\prime \prime}$. Although the resized covering balls may cover other $X$-points in $J_{n} \backslash J_{n}^{\prime \prime}$, the resized balls do not intersect $J_{n}^{\prime \prime \prime}$. The reason is that these balls can not contain the $Y$-points in the $s_{n} \times s_{n}$ squares. Specifically (but without loss of generality), suppose $Y_{j}$ is the $Y$-point closest to $X_{i}$, located at the position shown in Figure 2 (right). Then the radius of the resized covering ball $B\left(X_{i}\right)$ is $\sqrt{c^{2}+d^{2}}$, where the two segments with respective lengths $c$ and $d$ are also shown in Figure 2 (right). Since $c \leq s_{n}$ and $d \leq s_{n}$, we have $\sqrt{c^{2}+d^{2}} \leq \sqrt{2} s_{n}$. Note that the distance from $X_{i}$ to the boundary of $J_{n}^{\prime \prime \prime}$ is greater or equal to $\sqrt{2} s_{n}$. Thus, $B\left(X_{i}\right)$ does not intersect $J_{n}^{\prime \prime \prime}$. Thus, resizing the constrained covering balls of $X$-points in $J_{n} \backslash J_{n}^{\prime \prime}$ will decrease $\bar{\Gamma}_{n}$ by at most the number of $X$-points in $J_{n} \backslash J_{n}^{\prime \prime \prime}$, i.e. $\Delta_{J_{n}} \leq N_{X}\left(J_{n} \backslash J_{n}^{\prime \prime \prime}\right)$.

By the arguments in the preceding paragraph

$$
P\left(\frac{\Delta_{J_{n}}}{\left|J_{n}\right|}>\epsilon\right) \leq P\left(N_{X}\left(J_{n} \backslash J_{n}^{\prime \prime \prime}\right)>\epsilon\left|J_{n}\right|\right)+P\left(F_{n}^{c}\right)
$$


and by the union bound (for the middle inequality below), for $n$ sufficiently large and $s_{n}=\sqrt{(2+\delta) \log (n) / \lambda_{Y}}$ for some $\delta \in(0,1)$

$$
P\left(F_{n}^{c}\right) \leq P\left(E_{n}^{c}\right) \leq \frac{4 n}{s_{n}} e^{-s_{n}^{2} \lambda_{Y}} \leq n e^{-(2+\delta) \log n}
$$

which is summable in $n$, but also $\mathbf{E}\left[N_{X}\left(J_{n} \backslash J_{n}^{\prime \prime \prime}\right)\right] \sim c n s_{n}$, so by a Chernoff bound for the Binomial distribution, for $n$ sufficiently large,

$$
P\left(N_{X}\left(J_{n} \backslash J_{n}^{\prime \prime \prime}\right)>\epsilon n^{2}\right) \leq e^{-n}
$$

which is also summable in $n$.

Notice that this choice of $s_{n}$ implies that $P\left(F_{n}^{c}\right) \rightarrow 0$ as $n \rightarrow \infty$. By the Borel-Cantelli Lemma, the calculation above immediately implies that $\frac{\Delta_{J_{n}}}{\left|J_{n}\right|} \stackrel{a . s}{\longrightarrow} 0$. Since $\frac{\Gamma_{J_{n}}}{\left|J_{n}\right|}=\frac{\bar{\Gamma}_{J_{n}}}{\left|J_{n}\right|}+\frac{\Delta_{J_{n}}}{\left|J_{n}\right|}$, and both limits on the right hand side exist a.s., $\lim _{n \rightarrow \infty} \frac{\Gamma J_{n}}{\left|J_{n}\right|}$ exists a.s. and

$$
\lim _{n \rightarrow \infty} \frac{\Gamma_{J_{n}}}{\left|J_{n}\right|}=\lim _{n \rightarrow \infty} \frac{\bar{\Gamma}_{J_{n}}}{\left|J_{n}\right|} \text { a.s. }
$$

However, to prove Theorem 1.6, we need to show that the result of the above Lemma also holds for $\Gamma_{J_{t}}$ for real $t$.

Proof of Theorem 1.6. We first define $\Delta_{J_{t}}=\bar{\Gamma}_{J_{n}}-\Gamma_{J_{t}}$, for any $t \in[n, n+1)$. Note that $\Delta_{J_{n}}$ defined before is the difference between the two processes for the same region $J_{n}$, whereas $\Delta_{J_{t}}$ defined above is the difference over two different regions, $J_{n}$ and $J_{t}$. It is possible that $\Gamma_{J_{t}}>\bar{\Gamma}_{J_{n}}$ (i.e., $\Delta_{J_{t}}<0$ ), but $\Gamma_{J_{t}}$ can only be larger than $\bar{\Gamma}_{J_{n}}$ by at most $N_{X}\left(J_{t} \backslash J_{n}\right)$, the number of $X$-points in $J_{t} \backslash J_{n}$. Therefore, we obtain the following lower bound for $\Delta_{J_{t}}$ :

$$
\triangle_{J_{t}} \geq-N_{X}\left(J_{t} \backslash J_{n}\right) \geq-N_{X}\left(J_{n+1} \backslash J_{n}\right) .
$$

Below we will show that

$$
\frac{\Delta_{J_{t}}}{\left|J_{t}\right|} \geq \frac{-N_{X}\left(J_{n+1} \backslash J_{n}\right)}{\left|J_{t}\right|} \stackrel{a . s}{\longrightarrow} 0
$$

On the other hand, recall that $F_{n}$ is the event that all constrained covering balls of $X$-points in $J_{n}^{\prime \prime}$ are contained in $J_{n}$. Then given $F_{n}$, the covering balls of $X$-points in $J_{n}^{\prime \prime}$ are completely contained in $J_{n}$, so by the same argument for $\Gamma_{J_{n}}$, we know that $\bar{\Gamma}_{J_{n}}$ can be larger than $\Gamma_{J_{t}}$ by no more than the number of $X$-points in $J_{t} \backslash J_{n}^{\prime \prime \prime}$, thus we have

$$
\Delta_{J_{t}} /\left|J_{t}\right| \leq N_{X}\left(J_{t} \backslash J_{n}^{\prime \prime \prime}\right) /\left|J_{t}\right|
$$

Convergence to zero for the lower and upper bounds of $\Delta_{J_{t}} /\left|J_{t}\right|$ would yield the result of Theorem 1.6.

We will prove Theorem 1.6 by showing $\limsup _{t \rightarrow \infty} \frac{\Gamma_{J_{t}}}{\left|J_{t}\right|} \leq \lim _{n \rightarrow \infty} \frac{\bar{\Gamma}_{J_{n}}}{\left|J_{n}\right|}$ and $\liminf _{t \rightarrow \infty} \frac{\Gamma_{J_{t}}}{\left|J_{t}\right|} \geq \lim _{n \rightarrow \infty} \frac{\bar{\Gamma}_{J_{n}}}{\left|J_{n}\right|}$. We prove the convergence of the upper bound first. 
For any $t>0$, there is an integer $n(t)$ such that $n(t) \leq t<n(t)+1$. By the definitions above, $\Gamma_{J_{t}}=$ $\bar{\Gamma}_{J_{n(t)}}-\triangle_{J_{t}}$. In addition, we have shown that $\Delta_{J_{t}} \geq-N_{X}\left(J_{n(t)+1} \backslash J_{n(t)}\right)$, so

$$
\frac{\Gamma_{J_{t}}}{\left|J_{t}\right|} \leq \frac{\bar{\Gamma}_{J_{n(t)}}-\Delta_{J_{t}}}{\left|J_{n(t)}\right|} \leq \frac{\bar{\Gamma}_{J_{n(t)}}}{\left|J_{n(t)}\right|}+\frac{N_{X}\left(J_{n(t)+1} \backslash J_{n(t)}\right)}{\left|J_{n(t)}\right|}
$$

It should also be noted that

$$
\frac{N_{X}\left(J_{n(t)+1} \backslash J_{n(t)}\right)}{\left|J_{n(t)}\right|}=\frac{N_{X}\left(J_{n(t)+1}\right)}{\left|J_{n(t)+1}\right|} \cdot \frac{\left|J_{n(t)+1}\right|}{\left|J_{n(t)}\right|}-\frac{N_{X}\left(J_{n(t)}\right)}{\left|J_{n(t)}\right|} \stackrel{a . s}{\longrightarrow} \lambda_{X} \cdot 1-\lambda_{X}=0 .
$$

Therefore,

$$
\limsup _{t \rightarrow \infty} \frac{\Gamma_{J_{t}}}{\left|J_{t}\right|} \leq \lim _{n \rightarrow \infty} \frac{\bar{\Gamma}_{J_{n}}}{\left|J_{n}\right|} \text { a.s. }
$$

For the other direction, we first write

$$
\frac{\Gamma_{J_{t}}}{\left|J_{t}\right|}=\frac{\Gamma_{J_{t}}}{\left|J_{t}\right|} \cdot \mathbf{I}_{F_{n(t)}}+\frac{\Gamma_{J_{t}}}{\left|J_{t}\right|} \cdot \mathbf{I}_{F_{n(t)}^{c}}
$$

where $\mathbf{I}_{A}$ represents the indicator function for the event or set $A$.

Applying the same technique as when we showed $\Delta_{J_{n}} \leq N_{X}\left(J_{n} \backslash J_{n}^{\prime \prime \prime}\right)$, given $F_{n(t)}$, we see that when the boundary constraint is ignored, the constrained covering balls centered at $X$-points in $J_{n(t)}^{\prime \prime}$ do not change, whereas the covering balls centered at $X$-points in $J_{t} \backslash J_{n(t)}^{\prime \prime}$ do not intersect with $J_{n(t)}^{\prime \prime \prime}$. Therefore, we conclude that $\Delta_{J_{t}} \leq N_{X}\left(J_{t} \backslash J_{n(t)}^{\prime \prime \prime}\right)$. Hence, for the first term on the right hand side of equation (3.2), we have

$$
\frac{\Gamma_{J_{t}}}{\left|J_{t}\right|} \cdot \mathbf{I}_{F_{n(t)}} \geq \frac{\bar{\Gamma}_{J_{n(t)}}-\Delta_{J_{t}}}{\left|J_{n(t)+1}\right|} \cdot \mathbf{I}_{F_{n(t)}} \geq\left(\frac{\bar{\Gamma}_{J_{n(t)}}}{\left|J_{n(t)+1}\right|}-\frac{N_{X}\left(J_{t} \backslash J_{n(t)}^{\prime \prime \prime}\right)}{\left|J_{n(t)+1}\right|}\right) \cdot \mathbf{I}_{F_{n(t)}}
$$

Recall that we have chosen $s_{n(t)}=\sqrt{(2+\delta) \log (n(t)) / \lambda_{Y}}$. Because we have shown that $P\left(F_{n(t)}^{c}\right)=$ $o\left(\frac{1}{n(t)^{1+\delta}}\right)$, the Borel-Cantelli Lemma gives $\mathbf{I}_{F_{n(t)}^{c}} \stackrel{a . s}{\longrightarrow}$ 0. Moreover, we have

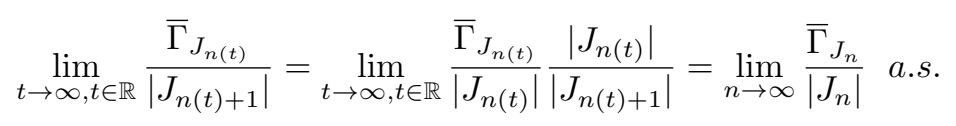

and

$$
\frac{N_{X}\left(J_{t} \backslash J_{n(t)}^{\prime \prime \prime}\right)}{\left|J_{n(t)+1}\right|}=\frac{N_{X}\left(J_{t}\right)}{\left|J_{t}\right|} \cdot \frac{\left|J_{t}\right|}{\left|J_{n(t)+1}\right|}-\frac{N_{X}\left(J_{n(t)}^{\prime \prime \prime}\right)}{\left|J_{n(t)}^{\prime \prime \prime}\right|} \cdot \frac{\left|J_{n(t)}^{\prime \prime \prime}\right|}{\left|J_{n(t)+1}\right|} \stackrel{a . s}{\longrightarrow} \lambda_{X} \cdot 1-\lambda_{X} \cdot 1=0,
$$


since $\frac{\left|J_{n(t)}^{\prime \prime \prime}\right|}{\left|J_{n(t)+1}\right|} \rightarrow 1$ as $n \rightarrow \infty$ by the choice of $s_{n}$. Thus, substituting the expressions above into Inequality (3.3), we immediately get

$$
\liminf _{t \rightarrow \infty} \frac{\Gamma_{J_{t}}}{\left|J_{t}\right|} \cdot \mathbf{I}_{F_{n(t)}} \geq \lim _{n \rightarrow \infty} \frac{\bar{\Gamma}_{J_{n}}}{\left|J_{n}\right|} \text { a.s. }
$$

In addition, note that $\frac{\Gamma_{J_{t}}}{\left|J_{t}\right|} \leq \frac{N_{X}\left(J_{t}\right)}{\left|J_{t}\right|} \stackrel{a . s}{\longrightarrow} \lambda_{X}$. Also, recall that, since $\mathbf{I}_{F_{n(t)}^{c}} \stackrel{a . s}{\longrightarrow} 0$,

$$
\frac{\Gamma_{J_{t}}}{\left|J_{t}\right|} \cdot \mathbf{I}_{F_{n(t)}^{c}} \stackrel{a . s}{\longrightarrow} 0 .
$$

Therefore, we can incorporate the two results above into equation (3.2) to get

$$
\liminf _{t \rightarrow \infty} \frac{\Gamma_{J_{t}}}{\left|J_{t}\right|} \geq \lim _{n \rightarrow \infty} \frac{\bar{\Gamma}_{J_{n}}}{\left|J_{n}\right|} \text { a.s. }
$$

Furthermore, combining Inequalities (3.1) and (3.4), we conclude that

$$
\lim _{t \rightarrow \infty, t \in \mathbb{R}} \frac{\Gamma_{J_{t}}}{\left|J_{t}\right|}=\lim _{n \rightarrow \infty} \frac{\bar{\Gamma}_{J_{n}}}{\left|J_{n}\right|} \text { a.s. }
$$

Thus, since $\left\{\frac{\bar{\Gamma}_{J_{n}}}{\left|J_{n}\right|}\right\}$ is a subsequence of $\left\{\frac{\bar{\Gamma}_{J_{q}}}{\left|J_{q}\right|}\right\}$, it follows that $\lim _{n \rightarrow \infty} \frac{\bar{\Gamma}_{J_{n}}}{\left|J_{n}\right|}=\lim _{q \rightarrow \infty, q \in \mathbb{Q}} \frac{\bar{\Gamma}_{J_{q}}}{\left|J_{q}\right|}$ a.s., where the existence of the latter is guaranteed as shown in the Proof of Theorem 1.5. That is, $\lim _{t \rightarrow \infty, t \in \mathbb{R}} \frac{\Gamma_{J_{t}}}{\left|J_{t}\right|}=\lim _{n \rightarrow \infty} \frac{\bar{\Gamma}_{J_{n}}}{\left|J_{n}\right|}=g_{2}(r)$ a.s. where $g_{2}(r)$ is as in Theorem 1.5.

\section{WLLN FOR THE DOMINATION NUMBER OF CCCDS GENERATED BY UNIFORM DATA IN $[0,1]^{2}$}

In the previous section, we established the SLLN for the domination number generated by the Poisson points in $\mathbb{R}^{2}$. In this section, we show the WLLN for the domination number for uniform data sets in $[0,1]^{2}$ (i.e., prove Thm. 1.7) by transferring the result in the Poisson case to the uniform distribution case.

Proof of Theorem 1.7. In the Poisson case, without loss of generality, we let the rates be $\lambda_{X}=1$ and $\lambda_{Y}=r$. For any integer $n>0$, we let $T(n)$ be the smallest real number such that there are $n+1 X$-points in the closure of $J_{T(n)}$. Note that the $(n+1)$-st $X$-point is on the boundary of $J_{T(n)}$, and the other $n X$-points are in the interior of $J_{T(n)}$. Moreover, by the SLLN, $N_{X}\left(J_{t}\right) /\left|J_{t}\right| \rightarrow 1$ as $t \rightarrow \infty$ through the real numbers a.s. Hence, taking $t=T(n)$, we have $n / J_{T(n)} \rightarrow 1$ a.s. Define $\Gamma_{n, M_{n}}=\Gamma_{J_{T(n)}}$, where $M_{n}$ is the (random) number of $Y$-points in $J_{T(n)}$. We know, by Theorem 1.6, that $\lim _{n \rightarrow \infty} \frac{\Gamma_{J_{T(n)}}\left|J_{T(n)}\right|}{J_{T}}=g_{2}(r)$ a.s., since $T(n) \rightarrow \infty$ a.s. as $n \rightarrow \infty$. Equivalently,

$$
\lim _{n \rightarrow \infty} \frac{\Gamma_{n, M_{n}}}{\left|J_{T(n)}\right|}=g_{2}(r) \text { a.s. }
$$

Combining the equation above with the fact that $\lim _{n \rightarrow \infty} \frac{n}{\left|J_{T(n)}\right|}=\lambda_{X}=1$ a.s., we obtain

$$
\lim _{n \rightarrow \infty} \frac{\Gamma_{n, M_{n}}}{n}=\lim _{n \rightarrow \infty} \frac{\Gamma_{n, M_{n}}}{n} \cdot \frac{n}{\left|J_{T(n)}\right|}=\lim _{n \rightarrow \infty} \frac{\Gamma_{n, M_{n}}}{\left|J_{T(n)}\right|}=g_{2}(r) \text { a.s. }
$$


Since almost sure convergence implies convergence in probability, it follows that

$$
\lim _{n \rightarrow \infty} \frac{\Gamma_{n, M_{n}}}{n}=g_{2}(r) \text { in probability }
$$

as well. From the conditional uniformity property of Poisson processes, the $n X$-points and $M_{n} Y$-points are both uniformly distributed in $J_{T(n)}$. Recall that the desired number of $Y$-points is $m=m(n)$, which is a nonrandom function of $n$. For simplicity, we will use $m$ instead of $m(n)$ in the rest of the proof. On the other hand, $M_{n}$ is the random number of $Y$-points in the Poisson case. If $M_{n}<m$, we will add $m-M_{n} Y$-points in the region $J_{T(n)}$ in a uniform way. Similarly, if $M_{n}>m$, then we will delete $M_{n}-m Y$-points uniformly from the $M_{n} Y$-points in $J_{T(n)}$. Of course, if $M_{n}=m$, no change is needed. After such modifications, the original $M_{n}$ $Y$-points become $m Y$-points. Let $\Gamma_{n, m}^{\prime}$ denote the domination number generated by the CCCD based on $n X$ points and the $m Y$-points, which are uniformly distributed in $J_{T(n)}$. Note that $\Gamma_{n, m}^{\prime}$ has the same distribution as $\Gamma_{n, m}$, the domination number of the CCCD based on $n X$-points and $m Y$-points uniformly distributed in $[0,1]^{2}$. Hence, if we can prove

$$
\lim _{n \rightarrow \infty} \frac{\Gamma_{n, m}^{\prime}}{n}=g_{2}(r) \text { in probability }
$$

then we have $\lim _{n \rightarrow \infty} \frac{\Gamma_{n, m}}{n}=\lim _{n \rightarrow \infty} \frac{\Gamma_{n, m}^{\prime}}{n}=g_{2}(r)$ in probability. Hence, $\lim _{n \rightarrow \infty} \frac{\Gamma_{n, m}}{n}=g_{2}(r)$ in probability, since the limit is a constant. So, the problem reduces to showing equation (4.2). In fact, if we let $\Delta_{n, M_{n}}=\Gamma_{n, m}^{\prime}-\Gamma_{n, M_{n}}$, and if we can prove

$$
\frac{\Delta_{n, M_{n}}}{n} \rightarrow 0 \text { in probability, }
$$

then using the result in (4.1), by Slutsky's Theorem, we obtain

$$
\lim _{n \rightarrow \infty} \frac{\Gamma_{n, m}^{\prime}}{n}=\lim _{n \rightarrow \infty} \frac{\Gamma_{n, M_{n}}}{n}+\lim _{n \rightarrow \infty} \frac{\Delta_{n, M_{n}}}{n}=g_{2}(r) \text { in probability. }
$$

All that remains from the discussion above is to prove the following lemma, the proof of which is provided in the Appendix.

Lemma 4.1. $\frac{\Delta_{n, M_{n}}}{n} \rightarrow 0$ in probability.

In this paper, the exact form of $g_{2}(r)$ is not identified. However, we can establish the following properties of $g_{2}(r)$ for which proofs are deferred to the Appendix as well.

Corollary 4.2. The limiting function $g_{2}(r)$ is a bounded, increasing, and continuous function of $r$ on $(0, \infty)$.

\section{WLLN FOR THE DOMINATION NUMBER OF CCCDS GENERATED BY NON-UNIFORM DATA IN $[0,1]^{2}$}

In this section, we provide the proof of Theorem 1.8 which establishes the WLLN for the domination number of CCCDs based on continuous and bounded densities in $[0,1]^{2}$. We proceed as in [31] where the SLLN for the domination number of CCCDs with continuous densities in one dimension were proved. In the following two subsections, we first generalize Theorem 1.7 to piece-wise constant densities, then extend it to the continuous case. The proofs are analogous to those in [31]. However, adding or deleting a point in two dimensions could change the domination number quite a bit (as large as $n-1$ ) whereas adding or deleting a point in one dimension can only change the domination number by at most 2 . But such large changes are very unlikely to happen, and their probabilities are proved to be negligible in the limit as $n \rightarrow \infty$. 


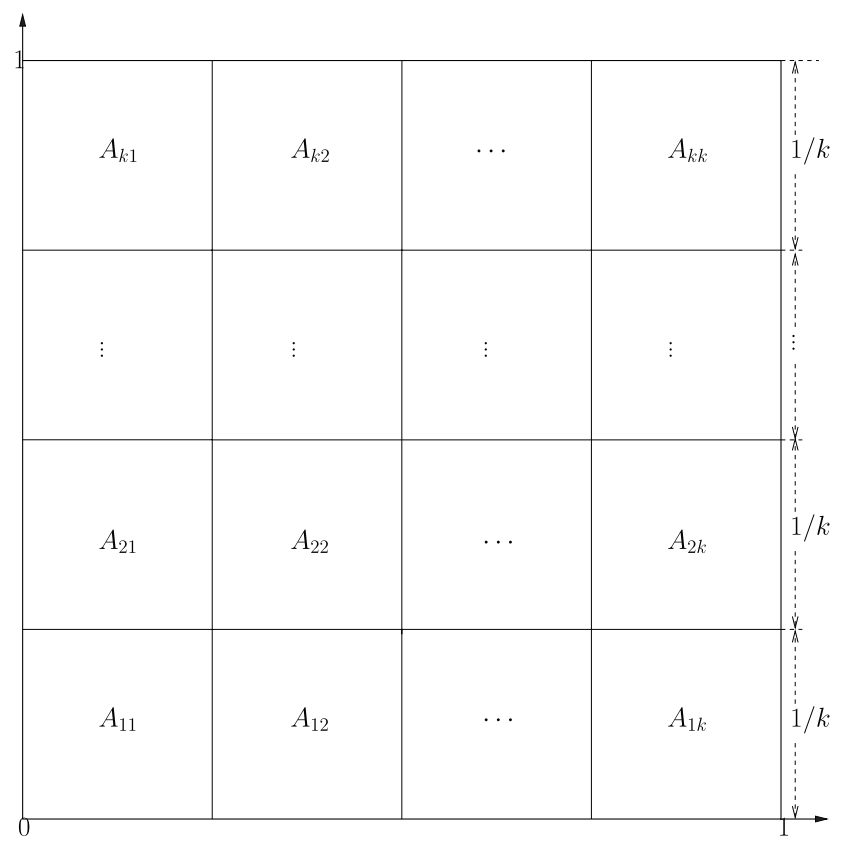

Figure 3 . The squares $A_{p q}$ on which densities $f_{X}$ and $f_{Y}$ are constant.

\subsection{The case of piecewise constant densities}

We consider the simple situation in which $f_{X}$ and $f_{Y}$ are piecewise constant densities defined as

$$
f_{X}(u, v)=\sum_{p, q=1}^{k} a_{p q} \mathbf{I}_{A_{p q}}(u, v) \quad \text { and } \quad f_{Y}(u, v)=\sum_{p, q=1}^{k} b_{p q} \mathbf{I}_{A_{p q}}(u, v)
$$

where $A_{p q}, p, q=1,2, \ldots, k$ equally divide $[0,1]^{2}$ into $k^{2}$ squares (see Fig. 3) and $a_{p q}>0$ and $b_{p q}>0$ for all $p, q$. Note that $\sum_{p, q=1}^{k} a_{p q}=\sum_{p, q=1}^{k} b_{p q}=k^{2}$.

Let $\Gamma_{n, m}$ be the domination number generated by the $n X$-points and $m Y$-points from $f_{X}$ and $f_{Y}$, respectively, in $[0,1]^{2}$, and $\Gamma_{n_{p q}, m_{p q}}$ be the domination number generated by the $n_{p q} X$-points and $m_{p q} Y$-points in $A_{p q}$. One can think of $\sum_{p, q} \Gamma_{n_{p q}, m_{p q}}$ as a "filtered" domination number generated by adding a "filter" $A_{p q}$ for each $\Gamma_{n_{p q}, m_{p q}}$. The effect of adding a filter is that no points outside $A_{p q}$ contribute to $\Gamma_{n_{p q}, m_{p q}}$. The outcome of ignoring the filters is the restoration of the sum of the "filtered" domination numbers $\sum_{p, q} \Gamma_{n_{p q}, m_{p q}}$ to the domination number $\Gamma_{n, m}$.

Lemma 5.1. $\sum_{p, q} \frac{\Gamma_{n_{p q}, m_{p q}}}{n} \rightarrow \mathcal{L}_{2}\left(r, f_{X}, f_{Y}\right)$ in probability, where $\mathcal{L}_{2}\left(r, f_{X}, f_{Y}\right)$ is as in equation (1.1).

Proof of Lemma 5.1. By the SLLN (see Lemma 2 of [31] for more details in the $d=1$ case), it follows that as $n \rightarrow \infty$,

$$
\frac{n_{p q}}{n} \rightarrow a_{p q}\left|A_{p q}\right|=\frac{a_{p q}}{k^{2}} \quad \text { a.s. } \quad \text { and } \quad \frac{m_{p q}}{m} \rightarrow b_{p q}\left|A_{p q}\right|=\frac{b_{p q}}{k^{2}} \quad \text { a.s. }
$$


Hence, $m_{p q} / n_{p q} \rightarrow r_{p q}$ a.s., where $r_{p q}=r \cdot b_{p q} / a_{p q}$. Therefore, applying Theorem 1.7 on each $A_{p q}$ yields

$$
\frac{\Gamma_{n_{p q}, m_{p q}}}{n}=\frac{\Gamma_{n_{p q}, m_{p q}}}{n_{p q}} \frac{n_{p q}}{n} \rightarrow g_{2}\left(r_{p q}\right) \cdot a_{p q}\left|A_{p q}\right| \text { in probability, }
$$

and thus

$$
\sum_{p, q} \frac{\Gamma_{n_{p q}, m_{p q}}}{n}=\sum_{p, q} \frac{\Gamma_{n_{p q}, m_{p q}}}{n_{p q}} \frac{n_{p q}}{n} \rightarrow \sum_{p, q} g_{2}\left(r_{p q}\right) \cdot a_{p q}\left|A_{p q}\right| \text { in probability. }
$$

Writing the above expression in the form of an integral gives

$$
\sum_{p, q} \frac{\Gamma_{n_{p q}, m_{p q}}}{n} \rightarrow \sum_{p, q} g_{2}\left(r \cdot b_{p q} / a_{p q}\right) \cdot a_{p q}\left|A_{p q}\right|=\mathcal{L}_{2}\left(r, f_{X}, f_{Y}\right) \text { in probability }
$$

where $f_{X}$ and $f_{Y}$ are as in equation (5.1).

Remark 5.2. The proof above can be easily generalized to the case when the regions of constancy for the densities are rectangles instead of squares. However, the limiting function $g_{2}$, then depends on the ratio between the length and the width of the rectangles, hence the final limiting value can not be written in a simple integral form, hence the square partition of the unit square.

Lemma 5.3. Let $\Delta_{n, m}=\Gamma_{n, m}-\sum_{p, q} \Gamma_{n_{p q}, m_{p q}}$. Then as $n \rightarrow \infty, \frac{\Delta_{n, m}}{n} \rightarrow 0$ in probability.

Proof. We prove this lemma by applying the same technique used in Section 2 . Specifically, with $\nu=\nu(n)$ to be chosen later, we shrink each $A_{p q}$ additively by $\xi=1 / k \nu$ to get $A_{p q}^{\prime}$, then shrink $A_{p q}^{\prime}$ additively by $\xi$ to get $A_{p q}^{\prime \prime}$, and then shrink $A_{p q}^{\prime \prime}$ additively by $\sqrt{2} \xi$ to get $A_{p q}^{\prime \prime \prime}$ (see Fig. 4). Finally, we divide $A_{p q}^{\prime} \backslash A_{p q}^{\prime \prime}$ equally into $4 \nu-12$ small squares with side length $\xi$. Then, there are totally $(4 \nu-12) k^{2}$ small squares in $\cup A_{p, q}=[0,1]^{2}$.

Define the event $E_{m}:=\{\exists$ at least one $Y$-point in each small square $\}$. To analyze the probability of the event $E_{m}$, we will consider the complementary event. First consider one particular square $A_{p q}$ and the event $E_{p q}$ that there is no $Y$-point in a small square in $A_{p q}$. Let $b^{*}=\min _{p, q}\left\{b_{p q}\right\}$, and require that $\xi \leq 1 / \sqrt{b^{*}}$ to make the second expression in equation (5.3) below positive. Since all $m Y$-points must fall outside $A_{p q}$, we have

$$
P\left(E_{p q}\right)=\left(1-\xi^{2} b_{p q}\right)^{m} \leq e^{-\xi^{2} b_{p q} m}
$$

By Boole's inequality, we have

$$
P\left(E_{m}^{c}\right)=P\left(\bigcup_{p, q} E_{p q}\right) \leq \sum_{p, q} e^{-\xi^{2} b_{p q} m} \leq(4 \nu-12) k^{2} e^{-\xi^{2} b^{*} m} \leq 4 \nu k^{2} e^{-\xi^{2} b^{*} m}
$$

Next, we apply the results obtained in the proof of the SLLN of the domination number in the Poisson case (refer to Fig. 2). Conditional on $E_{m}$, the covering ball of any $X$-point in $A_{p q}^{\prime \prime}$ is contained in $A_{p q}$. Therefore, ignoring the filter $A_{p q}$ has no effect on these $X$-points. However, there may be some $Y$-points just outside the boundary of $A_{p q}$, while some $X$-point in $A_{p q} \backslash A_{p q}^{\prime \prime}$ could have a covering ball that is not contained in $A_{p q}$. Thus, ignoring the filter $A_{p q}$ could reduce the covering ball of some $X$-points in $A_{p q} \backslash A_{p q}^{\prime \prime}$, thereby increasing the domination number. Such an increase is bounded by the number of $X$-points in $A_{p q} \backslash A_{p q}^{\prime \prime \prime}$, since no covering ball of any $X$-point in $A_{p q} \backslash A_{p q}^{\prime \prime}$ can intersect with $A_{p q}^{\prime \prime \prime}$. Summarizing the argument above, we obtain

$$
\frac{\Delta_{n, m}}{n}=\frac{\Delta_{n, m}}{n} \mathbf{I}_{E_{m}}+\frac{\Delta_{n, m}}{n} \mathbf{I}_{E_{m}^{c}}
$$




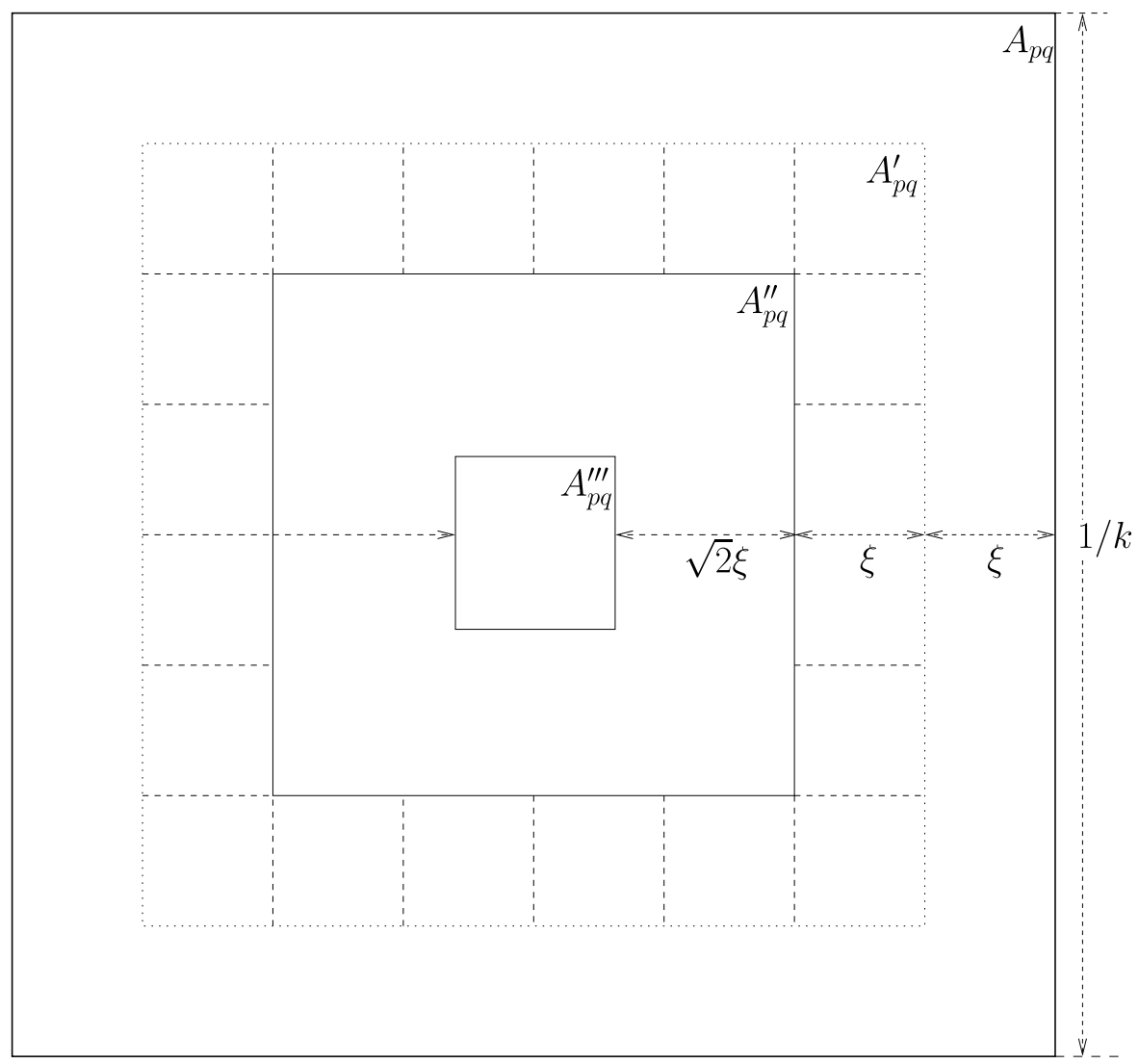

Figure 4 . The squares $A_{p q}, A_{p q}^{\prime}, A_{p q}^{\prime \prime}$ and $A_{p q}^{\prime \prime \prime}$.

where

$$
\left|\frac{\Delta_{n, m}}{n} \mathbf{I}_{E_{m}^{c}}\right| \leq \mathbf{I}_{E_{m}^{c}}
$$

and

$$
0 \leq \frac{\Delta_{n, m}}{n} \mathbf{I}_{E_{m}} \leq \frac{\sum_{p, q} N_{X}\left(A_{p q} \backslash A_{p q}^{\prime \prime \prime}\right)}{n}
$$

We may choose the relationships between the parameters. Let $\nu=\left\lfloor\sqrt{\frac{m b^{*}}{k^{4} \cdot \log \sqrt{m}}}\right\rfloor$ and in order to satisfy $\xi \leq 1 / \sqrt{b^{*}}$, we need to take $k \leq \sqrt{m} / \sqrt{\log \sqrt{m}}$. For sufficiently large $k$ and $m$ with $k$ being no larger than $\sqrt{m} / \sqrt{\log \sqrt{m}}$, we have

$$
P\left(E_{m}^{c}\right) \leq 4 \nu k^{2} \exp \left(-\frac{m b^{*}}{k^{2} \nu^{2}}\right) \leq \frac{4 \sqrt{b^{*}}}{\sqrt{m^{k^{2}-1} \log \sqrt{m}}} \rightarrow 0 \quad \text { as } m \rightarrow \infty
$$


so $\mathbf{I}_{E_{m}^{c}} \rightarrow 0$ in probability. The expression above combined with Inequality (5.5) yields

$$
\frac{\Delta_{n, m}}{n} \mathbf{I}_{E_{m}^{c}} \rightarrow 0 \text { in probability. }
$$

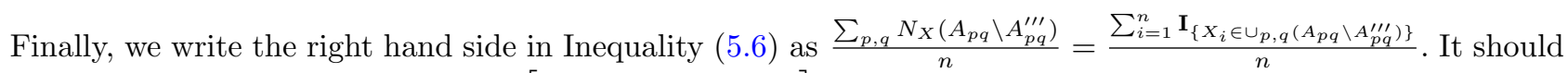
be noted that (see Figs. 3 and 4.) $\mathbf{E}\left[\mathbf{I}_{\left\{X_{i} \in \cup_{p, q}\left(A_{p q} \backslash A_{p q}^{\prime \prime \prime}\right)\right\}}\right]=P\left(X_{i} \in \cup_{p, q}\left(A_{p q} \backslash A_{p q}^{\prime \prime \prime}\right)\right)$ and

$$
P\left(X_{i} \in A_{p q} \backslash A_{p q}^{\prime \prime \prime}\right)=a_{p q}\left(\frac{1}{k^{2}}-\left(\frac{1}{k}-2(2+\sqrt{2}) \xi\right)^{2}\right)=a_{p q}\left(\frac{4(2+\sqrt{2}) \xi}{k}-4(2+\sqrt{2})^{2} \xi^{2}\right) .
$$

So,

$$
\begin{aligned}
P\left(X_{i} \in \cup_{p, q}\left(A_{p q} \backslash A_{p q}^{\prime \prime \prime}\right)\right)=\sum_{p, q} & a_{p q}\left(\frac{4(2+\sqrt{2}) \xi}{k}-4(2+\sqrt{2})^{2} \xi^{2}\right) \\
& =k^{2}\left(\frac{4(2+\sqrt{2}) \xi}{k}-4(2+\sqrt{2})^{2} \xi^{2}\right)=4(2+\sqrt{2}) \frac{1}{\nu}-4(2+\sqrt{2})^{2} \frac{1}{\nu^{2}} .
\end{aligned}
$$

Since $1 / \nu \rightarrow 0$ as $m \rightarrow \infty$, we have

$$
\mathbf{E}\left[\mathbf{I}_{\left\{X_{i} \in \cup_{p, q}\left(A_{p q} \backslash A_{p q}^{\prime \prime \prime}\right)\right\}}\right]=4(2+\sqrt{2}) \frac{1}{\nu}-4(2+\sqrt{2})^{2} \frac{1}{\nu^{2}} \longrightarrow 0 \text { as } m \rightarrow \infty .
$$

Therefore, for any $\delta>0$, the Markov inequality provides

$$
P\left(\frac{\sum_{i=1}^{n} \mathbf{I}_{\left\{X_{i} \in \cup_{p, q}\left(A_{p q} \backslash A_{p q}^{\prime \prime \prime}\right)\right\}}}{n} \geq \delta\right) \leq \frac{\mathbf{E}\left[\mathbf{I}_{\left\{X_{i} \in \cup_{p, q}\left(A_{p q} \backslash A_{p q}^{\prime \prime \prime}\right)\right\}}\right]}{\delta} \longrightarrow 0 \text { as } n \rightarrow \infty,
$$

and thus $\frac{\sum_{p, q} N_{X}\left(A_{p q} \backslash A_{p q}^{\prime \prime \prime}\right)}{n} \rightarrow 0$ in probability. Combining this with Inequality (5.6) yields

$$
\frac{\Delta_{n, m}}{n} \mathbf{I}_{E_{m}} \rightarrow 0 \text { in probability. }
$$

Finally, substituting equations (5.7) and (5.8) into equation (5.4) yields the desired result.

\subsection{The case of continuous bounded densities: Proof of Theorem 1.8}

If $f_{X}$ and $f_{Y}$ are bounded and continuous, then they are both uniformly continuous on $[0,1]^{2}$. Thus, given any $\delta>0$, there exists an integer $k_{0}$ such that for any $k \geq k_{0}$ and the equal partition $\left\{A_{p q}: p, q=1,2, \ldots, k\right\}$ of $[0,1]^{2}$ (see Fig. 3), the following must hold:

$$
\left|f_{X}\left(u_{1}, v_{1}\right)-f_{X}\left(u_{2}, v_{2}\right)\right| \leq \delta \quad \text { and } \quad\left|f_{Y}\left(u_{1}, v_{1}\right)-f_{Y}\left(u_{2}, v_{2}\right)\right| \leq \delta
$$

for any $\left(u_{1}, v_{1}\right),\left(u_{2}, v_{2}\right) \in A_{p q}$. We define piecewise constant functions approximating $f_{X}$ and $f_{Y}$ as follows: For $(u, v) \in A_{p q}$, define

$$
\bar{f}_{X}(u, v):=\min \left\{f_{X}(x, y),(x, y) \in A_{p q}\right\} \text { and } \bar{f}_{Y}(u, v):=\min \left\{f_{Y}(x, y),(x, y) \in A_{p q}\right\},
$$


and then rescale $\bar{f}_{X}$ and $\bar{f}_{Y}$ by dividing them by their respective integrals to give piecewise constant densities $\hat{f}_{X}$ and $\hat{f}_{Y}$, which approximate $f_{X}$ and $f_{Y}$, respectively.

For random vectors $U_{i}:=\left(X_{i 1}, X_{i 2}, X_{i 3}\right), i=1,2, \ldots, n$, distributed i.i.d. uniformly between $\{(u, v, 0)$ : $\left.(u, v) \in[0,1]^{2}\right\}$ and the surface $\left\{\left(u, v, f_{X}(u, v)\right):(u, v) \in[0,1]^{2}\right\}$, the marginal density of $X_{i}=\left(X_{i 1}, X_{i 2}\right)$ is proved to be $f_{X}$. A similar procedure generates i.i.d. random vectors $V_{j}:=\left(Y_{j 1}, Y_{j 2}, Y_{j 3}\right), j=1,2, \ldots, m$, with the marginal density of $Y_{j}=\left(Y_{j 1}, Y_{j 2}\right)$ being $f_{Y}$.

Next, we let $\bar{U}_{i}:=\left(\bar{X}_{i 1}, \bar{X}_{i 2}, \bar{X}_{i 3}\right), i=1,2, \ldots, n$, and $\bar{V}_{j}:=\left(\bar{Y}_{j 1}, \bar{Y}_{j 2}, \bar{Y}_{j 3}\right), j=1,2, \ldots, m$, be i.i.d. random vectors uniformly distributed under surfaces $\left\{\left(u, v, \bar{f}_{X}(u, v)\right):(u, v) \in[0,1]^{2}\right\}$ and $\left\{\left(u, v, \bar{f}_{Y}(u, v)\right)\right.$ : $\left.(u, v) \in[0,1]^{2}\right\}$, respectively. Moreover, $\bar{U}_{i}$ 's are independent of $U_{i}$ 's and $\bar{V}_{i}$ 's are independent of $V_{j}$ 's.

Finally, let $\bar{R}_{X}$ be the region between the surfaces

$$
\left\{\left(u, v, f_{X}(u, v)\right):(u, v) \in[0,1]^{2}\right\} \text { and }\left\{\left(u, v, \bar{f}_{X}(u, v)\right):(u, v) \in[0,1]^{2}\right\}
$$

and let $\bar{R}_{Y}$ be the region between the surfaces

$$
\left\{\left(u, v, f_{Y}(u, v)\right):(u, v) \in[0,1]^{2}\right\} \text { and }\left\{\left(u, v, \bar{f}_{Y}(u, v)\right):(u, v) \in[0,1]^{2}\right\} .
$$

We then define

$$
\widehat{U}_{i}:=\left(\widehat{X}_{i 1}, \widehat{X}_{i 2}, \widehat{X}_{i 3}\right)=U_{i} \mathbf{I}_{\left\{U_{i} \notin \bar{R}_{X}\right\}}+\bar{U}_{i} \mathbf{I}_{\left\{U_{i} \in \bar{R}_{X}\right\}} \text { for } i=1,2, \ldots, n
$$

and

$$
\widehat{V}_{j}:=\left(\widehat{Y}_{j 1}, \widehat{Y}_{j 2}, \widehat{Y}_{j 3}\right)=V_{j} \mathbf{I}_{\left\{V_{j} \notin \bar{R}_{Y}\right\}}+\bar{V}_{j} \mathbf{I}_{\left\{V_{j} \in \bar{R}_{Y}\right\}} \text { for } j=1,2, \ldots, m
$$

The sequences above can be interpreted as follows. For each $i \in[n]:=\{1,2, \ldots, n\}$, if the point $U_{i}=$ $\left(X_{i 1}, X_{i 2}, X_{i 3}\right)$ does not fall into $\bar{R}_{X}$, then this point is assigned to $\widehat{U}_{i}$; otherwise, $\bar{U}_{i}=\left(\bar{X}_{i 1}, \bar{X}_{i 2}, \bar{X}_{i 3}\right)$ is assigned to $\widehat{U}_{i}$. A similar procedure applies to $Y$-points. Again, using a similar technique to one in [31] shows that $\left(\widehat{X}_{i 1}, \widehat{X}_{i 2}\right)$ and $\left(\widehat{Y}_{j 1}, \widehat{Y}_{j 2}\right)$ have piecewise constant densities $\hat{f}_{X}$ and $\hat{f}_{Y}$, respectively.

Define $\mathcal{X}_{n}=\left\{X_{i}=\left(X_{i 1}, X_{i 2}\right), i=1,2, \ldots, n\right\}$, and $\mathcal{Y}_{m}=\left\{Y_{j}=\left(Y_{j 1}, Y_{j 2}\right), j=1,2, \ldots, m\right\}$. Let $\Gamma\left(\mathcal{X}_{n}, \mathcal{Y}_{m}\right)$ denote the domination number generated by $\mathcal{X}_{n}$ and $\mathcal{Y}_{m}$. Similarly, let $\Gamma\left(\widehat{\mathcal{X}}_{n}, \widehat{\mathcal{Y}}_{m}\right)$ denote the domination number generated by $\widehat{\mathcal{X}}_{n}=\left\{\widehat{X}_{i}=\left(\widehat{X}_{i 1}, \widehat{X}_{i 2}\right), i=1,2, \ldots, n\right\}$, and $\widehat{\mathcal{Y}}_{m}=\left\{\widehat{Y}_{j}=\left(\widehat{Y}_{j 1}, \widehat{Y}_{j 2}\right), j=1,2, \ldots, m\right\}$. Note that only the points $U_{i} \in \bar{R}_{X}$ and $V_{j} \in \bar{R}_{Y}$ could cause a difference between $\Gamma\left(\mathcal{X}_{n}, \mathcal{Y}_{m}\right)$ and $\Gamma\left(\widehat{\mathcal{X}}_{n}, \widehat{\mathcal{Y}}_{m}\right)$. But such a difference could be as large as $n-1$. However, by applying the results obtained in the proof of Theorem 1.7 in Section 4, we next show that if the largest covering ball is small, then the difference is negligible in the limit.

Handling $U_{i}$ Points in $\bar{R}_{X}$ : When any $U_{i} \in \bar{R}_{X}$ is replaced by $\bar{U}_{i}$, it is equivalent to deleting $X_{i}=\left(X_{i 1}, X_{i 2}\right)$ from $\mathcal{X}_{n}$ and then putting $\bar{X}_{i}=\left(\bar{X}_{i 1}, \bar{X}_{i 2}\right)$ in $\mathcal{X}_{n}$. Deleting $X_{i}$ could decrease (but never increase) the domination number $\Gamma\left(\mathcal{X}_{n}, \mathcal{Y}_{m}\right)$ by at most 1 , provided that $X_{i}$ is not the center of a covering ball. On the other hand, note that deleting the covering ball of $X_{i}$ could also increase (but never decrease) the domination number by at most the number of $X$-points in $B\left(X_{i}\right) \backslash\left\{X_{i}\right\}$. Hence, deleting $X_{i}$ could change the domination number by at most the number of $X$-points in $B\left(X_{i}\right)$.

Similarly, adding $\bar{X}_{i}$ could further increase the domination number by at most 1 . However, note that adding the covering ball of $\bar{X}_{i}$ can also decrease the domination number by at most the number of $\widehat{X}$-points in $B\left(\bar{X}_{i}\right) \backslash\left\{\bar{X}_{i}\right\}$. Hence, adding $\bar{X}_{i}$ can change the domination number by at most the number of $\widehat{X}$-points in 
$B\left(\bar{X}_{i}\right)$. Therefore, replacing any $U_{i} \in \bar{R}_{X}$ by $\bar{X}_{i}$ could only change the domination number by at most

$$
N_{X}\left(B\left(X_{i}\right)\right)+N_{\widehat{X}}\left(B\left(\bar{X}_{i}\right)\right)
$$

where $N_{X}\left(B\left(X_{i}\right)\right):=\sum_{l=1}^{n} \mathbf{I}_{\left\{X_{l} \in B\left(X_{i}\right)\right\}}$ and $N_{\widehat{X}}\left(B\left(\bar{X}_{i}\right)\right):=\sum_{l=1}^{n} \mathbf{I}_{\left\{\widehat{X}_{l} \in B\left(\bar{X}_{i}\right)\right\}}$. Hence, the change caused by the set of points $X_{i}$ in $\bar{R}_{X}$ is bounded by

$$
\sum_{i=1}^{n} \mathbf{I}_{\left\{U_{i} \in \bar{R}_{X}\right\}}\left(N_{X}\left(B\left(X_{i}\right)\right)+N_{\widehat{X}}\left(B\left(\bar{X}_{i}\right)\right)\right)
$$

Given any $U_{i} \in \bar{R}_{X}$, we denote the radius of the covering ball $B\left(X_{i}\right)$ by $B_{i}$. For any fixed $U_{i} \in \bar{R}_{X}$, we can bound $P\left(B_{i}>b\right)$ as follows:

$$
P\left(B_{i}>b \mid U_{i}, U_{i} \in \bar{R}_{X}\right) \leq P\left(\text { there are no } Y \text {-points in the ball centered at } X_{i} \text { with radius } b\right)
$$

Recall that $f_{X}, \hat{f}_{X}, f_{Y}$ and $\hat{f}_{Y}$ are all strictly positive and bounded, so we can assume $k_{1} \leq f_{X} \leq k_{2}, k_{1} \leq$ $\hat{f}_{X} \leq k_{2}, k_{1} \leq f_{Y} \leq k_{2}$ and $k_{1} \leq \hat{f}_{Y} \leq k_{2}$ for some strictly positive constants $k_{1}, k_{2}$. Hence, the inequality above can be further bounded as $P\left(B_{i}>b \mid U_{i}, U_{i} \in \bar{R}_{X}\right) \leq\left(1-k_{1} \pi b^{2}\right)^{m}$. Since the bound above is uniform for any $U_{i} \in \bar{R}_{X}$, it follows that $P\left(B_{i}>b \mid U_{i} \in \bar{R}_{X}\right) \leq\left(1-k_{1} \pi b^{2}\right)^{m}$. Note that for any $l \in[n]_{-i}:=\{1,2, \ldots, i-$ $1, i+1,2, \ldots, n\}$, the random point $X_{l}$ is independent of $X_{i}$ and $Y_{j}, j=1,2, \ldots, m$. Therefore,

$$
\begin{aligned}
& \mathbf{E}\left[\mathbf{I}_{\left\{X_{l} \in B\left(X_{i}\right)\right\}} \mid U_{i} \in \bar{R}_{X}\right]=\mathbf{E}\left[\mathbf{E}\left[\mathbf{I}_{\left\{X_{l} \in B\left(X_{i}\right)\right\}} \mid U_{i} \in \bar{R}_{X}, B_{i}\right] \mid U_{i} \in \bar{R}_{X}\right] \\
& =\mathbf{E}\left[P\left(X_{l} \in B\left(X_{i}\right) \mid U_{i} \in \bar{R}_{X}, B_{i}\right) \mid U_{i} \in \bar{R}_{X}\right] \leq \mathbf{E}\left[k_{2} \pi B_{i}^{2} \mid U_{i} \in \bar{R}_{X}\right] .
\end{aligned}
$$

By applying the same technique as in the proof of Lemma 4.1, Case 2 in the Appendix, we can further bound the above expectation as $\mathbf{E}\left[\mathbf{I}_{\left\{X_{l} \in B\left(X_{i}\right)\right\}} \mid U_{i} \in \bar{R}_{X}\right] \leq \int\left(1-k_{1} \pi b^{2}\right)^{m} \mathrm{~d}\left(k_{1} \pi b^{2}\right) \leq \frac{1}{m+1}$. Since $m / n \rightarrow r$, when $n$ is sufficiently large, it follows that

$$
\mathbf{E}\left[\sum_{l=1}^{n} \mathbf{I}_{\left\{X_{l} \in B\left(X_{i}\right)\right\}} \mid U_{i} \in \bar{R}_{X}\right]=1+\mathbf{E}\left[\sum_{l \in[n]_{-i}} \mathbf{I}_{\left\{X_{l} \in B\left(X_{i}\right)\right\}} \mid U_{i} \in \bar{R}_{X}\right] \leq 1+\frac{n-1}{m+1} \leq K_{1},
$$

for some constant $K_{1}>0$.

Similarly, when $n$ is sufficiently large, $\mathbf{E}\left[\sum_{l=1}^{n} \mathbf{I}_{\left\{\widehat{X}_{l} \in B\left(\bar{X}_{i}\right)\right\}} \mid U_{i} \in \bar{R}_{X}\right] \leq K_{1}$. From the two inequalities above, we bound the expectation of equation (5.10) as follows:

$$
\begin{aligned}
& \mathbf{E}\left[\sum_{i=1}^{n} \mathbf{I}_{\left\{U_{i} \in \bar{R}_{X}\right\}}\left(N_{X}\left(B\left(X_{i}\right)\right)+N_{\widehat{X}}\left(B\left(\bar{X}_{i}\right)\right)\right)\right]=\sum_{i=1}^{n} \mathbf{E}\left[\mathbf{I}_{\left\{U_{i} \in \bar{R}_{X}\right\}}\left(N_{X}\left(B\left(X_{i}\right)\right)+N_{\widehat{X}}\left(B\left(\bar{X}_{i}\right)\right)\right)\right] \\
& =\sum_{i=1}^{n} \mathbf{E}\left[\left(N_{X}\left(B\left(X_{i}\right)\right)+N_{\widehat{X}}\left(B\left(\bar{X}_{i}\right)\right)\right) \mid U_{i} \in \bar{R}_{X}\right] P\left(U_{i} \in \bar{R}_{X}\right) \\
& \leq \sum_{i=1}^{n} 2 K_{1} P\left(U_{i} \in \bar{R}_{X}\right)=2 K_{1} \sum_{i=1}^{n} P\left(U_{i} \in \bar{R}_{X}\right) \leq 2 K_{1} \delta n,
\end{aligned}
$$


where $\delta$ is the uniform bound on the densities $f_{X}$ and $f_{Y}$ introduced in equation (5.9). In fact, $\delta$ can be taken to be $\delta=P\left(U_{i} \in \bar{R}_{X}\right)$.

Handling $V_{j}$ Points in $\bar{R}_{Y}$ : After replacing all $U_{i} \in \bar{R}_{X}$ by $\bar{U}_{i}$, the original domination number $\Gamma\left(\mathcal{X}_{n}, \mathcal{Y}_{m}\right)$ becomes $\Gamma\left(\widehat{\mathcal{X}}_{n}, \mathcal{Y}_{m}\right)$. Next, we consider the effect of replacing $V_{j}=\left(Y_{j 1}, Y_{j 2}, Y_{j 3}\right) \in \bar{R}_{Y}$ by $\bar{V}_{j}=\left(\bar{Y}_{j 1}, \bar{Y}_{j 2}, \bar{Y}_{j 3}\right)$, which is equivalent to deleting $Y_{j}=\left(Y_{j 1}, Y_{j 2}\right)$ and then adding $\bar{Y}_{j}=\left(\bar{Y}_{j 1}, \bar{Y}_{j 2}\right)$. After replacing all $V_{j} \in \bar{R}_{Y}$ by $\bar{V}_{j}$, the domination number $\Gamma\left(\widehat{\mathcal{X}}_{n}, \mathcal{Y}_{m}\right)$ becomes $\Gamma\left(\widehat{\mathcal{X}}_{n}, \widehat{\mathcal{Y}}_{m}\right)$. We have discussed the effect of deleting and adding $\mathcal{Y}_{m}$-points in the proof of Theorem 1.7 in Section 4. For all $Y_{j} \notin \bar{R}_{Y}$, refer to $Y_{j}$ as a $\mathcal{Y}_{m}^{\prime}$-point. For any $Y_{j} \in \bar{R}_{Y}$, define $B_{j}^{y}$ as the maximum radius of all balls that contain $Y_{j}$ but contain no $\mathcal{Y}_{m}^{\prime}$-points. Applying the arguments in the proof of Lemma 4.1 in the Appendix shows that deleting $Y_{j}$ could decrease (but never increase) $\Gamma\left(\widehat{\mathcal{X}}_{n}, \mathcal{Y}_{m}\right)$ by at most the number of $\widehat{X}$-points in the ball $B\left(Y_{j}\right)=B\left(Y_{j}, 2 B_{j}^{y}\right)$, centered at $Y_{j}$ with radius $2 B_{j}^{y}$.

Furthermore, for any $\overline{Y_{j}} \in \bar{R}_{Y}$, define $\bar{B}_{j}$ as the maximum radius of all balls that contain $\overline{Y_{j}}$ but contain no $\mathcal{Y}_{m^{-}}^{\prime}$ points. Similarly, applying the arguments in the proof of Lemma 4.1 in the Appendix shows that adding $\overline{Y_{j}}$ could further increase (but never decrease) $\Gamma\left(\widehat{\mathcal{X}}_{n}, \mathcal{Y}_{m}\right)$ by at most the number of $\widehat{X}$-points in $B\left(\overline{Y_{j}}\right)=B\left(\overline{Y_{j}}, 2 \bar{B}_{j}\right)$, centered at $\overline{Y_{j}}$ with radius $2 \bar{B}_{j}$. Thus, replacing any $V_{j} \in \bar{R}_{Y}$ by $\overline{Y_{j}}$ could further change the original domination number $\Gamma\left(\mathcal{X}_{n}, \mathcal{Y}_{m}\right)$ by no more than

$$
\sum_{j=1}^{m} \mathbf{I}_{\left\{V_{j} \in \bar{R}_{Y}\right\}}\left(N_{\widehat{X}}\left(B\left(Y_{j}\right)\right)+N_{\widehat{X}}\left(B\left(\overline{Y_{j}}\right)\right)\right)
$$

where $N_{\widehat{X}}\left(B\left(Y_{j}\right)\right):=\sum_{l=1}^{n} \mathbf{I}_{\left\{\widehat{X}_{l} \in B\left(Y_{j}\right)\right\}}$ and $N_{\widehat{X}}\left(B\left(\overline{Y_{j}}\right)\right):=\sum_{l=1}^{n} \mathbf{I}_{\left\{\widehat{X}_{l} \in B\left(\overline{Y_{j}}\right)\right\}}$.

Let $M_{R}$ denote the number of $V_{j} \in \bar{R}_{Y}$. Note that $M_{R}$ is a $\operatorname{Binomial}(m, \delta)$ random variable and is based on the region between the two surfaces $\left\{\left(u, v, f_{Y}(u, v)\right):(u, v) \in[0,1]^{2}\right\}$ and $\left\{\left(u, v, \bar{f}_{Y}(u, v)\right):(u, v) \in[0,1]^{2}\right\}$.

For any fixed $V_{j} \in \bar{R}_{Y}$, using the same argument (and recalling the small grid balls inscribed in the squares) in the proof of Lemma 4.1 Case 1 in the Appendix, we bound $P\left(B_{j}^{y}>b \mid Y_{j}, Y_{j} \in \bar{R}_{Y}, M_{R}\right)$ as follows:

$$
P\left(B_{j}^{y}>b \mid Y_{j}, Y_{j} \in \bar{R}_{Y}, M_{R}\right) \leq 64 \times P\left(\exists \text { no } \mathcal{Y}_{m}^{\prime} \text {-points in any grid ball }\right) \leq 64\left(1-k_{1} \pi(b / 4)^{2}\right)^{m-M_{R}} .
$$

Since the above bound is uniform for any $V_{j} \in \bar{R}_{Y}$, it follows that $P\left(B_{j}^{y}>b \mid Y_{j} \in \bar{R}_{Y}, M_{R}\right) \leq 64(1-$ $\left.k_{1} \pi(b / 4)^{2}\right)^{m-M_{R}}$.

Note that for any $l \in[n]$, the random point $\widehat{X}_{l}$ is independent of $Y_{j}, j=1,2, \ldots, m$. Therefore,

$$
\begin{aligned}
& \mathbf{E}\left[\mathbf{I}_{\left\{\widehat{X}_{l} \in B\left(Y_{j}\right)\right\}} \mid Y_{j} \in \bar{R}_{Y}, M_{R}\right]=\mathbf{E}\left[\mathbf{E}\left[\mathbf{I}_{\left\{\widehat{X}_{l} \in B\left(Y_{j}\right)\right\}} \mid Y_{j} \in \bar{R}_{Y}, M_{R}, B_{j}^{y}\right] \mid Y_{j} \in \bar{R}_{Y}, M_{R}\right] \\
= & \mathbf{E}\left[P\left(\widehat{X}_{l} \in B\left(Y_{j}\right) \mid Y_{j} \in \bar{R}_{Y}, M_{R}, B_{j}^{y}\right) \mid Y_{j} \in \bar{R}_{Y}, M_{R}\right] \leq \mathbf{E}\left[k_{1} \pi\left(2 B_{j}^{y}\right)^{2} \mid Y_{j} \in \bar{R}_{Y}, M_{R}\right] .
\end{aligned}
$$

By applying the same technique as in the proof of Lemma 4.1, Case 2 in the Appendix, we can further bound the above expression as follows

$$
\mathbf{E}\left[\mathbf{I}_{\left\{\widehat{X}_{l} \in B\left(Y_{j}\right)\right\}} \mid Y_{j} \in \bar{R}_{Y}, M_{R}\right] \leq \int 64\left(1-k_{1} \pi(b / 4)^{2}\right)^{m-M_{R}} \mathrm{~d}\left(k_{2} \pi(2 b)^{2}\right) .
$$


Hence,

$$
\mathbf{E}\left[N_{\widehat{X}}\left(B\left(Y_{j}\right)\right) \mid Y_{j} \in \bar{R}_{Y}, M_{R}\right] \leq n \cdot \int 64\left(1-k_{1} \pi(b / 4)^{2}\right)^{m-M_{R}} \mathrm{~d}\left(k_{2} \pi(2 b)^{2}\right) \leq n \cdot \frac{C}{m-M_{R}}
$$

for some constant $C>0$.

Since $m / n \rightarrow r$, when $n$ is sufficiently large, conditional on $M_{R} \leq 2 \delta m$, the inequality above yields

$$
\mathbf{E}\left[N_{\widehat{X}}\left(B\left(Y_{j}\right)\right) \mid Y_{j} \in \bar{R}_{Y}, M_{R} \leq 2 \delta m\right] \leq n \cdot \frac{C}{m-2 \delta m} \leq K_{2}
$$

for some constant $K_{2}>0$.

Furthermore, by applying the argument above to the case of adding $\overline{Y_{j}}$, we conclude that, when $n$ is sufficiently large,

$$
\mathbf{E}\left[N_{\widehat{X}}\left(B\left(\bar{Y}_{j}\right)\right) \mid Y_{j} \in \bar{R}_{Y}, M_{R} \leq 2 \delta m\right] \leq K_{2}
$$

Note that

$$
\begin{aligned}
& \mathbf{E}\left[\sum_{j=1}^{m} \mathbf{I}_{\left\{V_{j} \in \bar{R}_{Y}\right\}}\left(N_{\widehat{X}}\left(B\left(Y_{j}\right)\right)+N_{\widehat{X}}\left(B\left(\bar{Y}_{j}\right)\right)\right) \mid M_{R} \leq 2 \delta m\right] \\
& =\sum_{j=1}^{m} \mathbf{E}\left[\left(N_{\widehat{X}}\left(B\left(Y_{j}\right)\right)+N_{\widehat{X}}\left(B\left(\bar{Y}_{j}\right)\right)\right) \mid V_{j} \in \bar{R}_{Y}, M_{R} \leq 2 \delta m\right] \times P\left(V_{j} \in \bar{R}_{Y} \mid M_{R} \leq 2 \delta m\right) .
\end{aligned}
$$

Applying Inequalities (5.13) and (5.14) to the above equation, we obtain

$$
\begin{aligned}
\mathbf{E}\left[\sum_{j=1}^{m} \mathbf{I}_{\left\{V_{j} \in \bar{R}_{Y}\right\}} \sum_{l=1}^{n}\left(\mathbf{I}_{\left\{\widehat{X}_{l} \in B\left(Y_{j}\right)\right\}}+\mathbf{I}_{\left\{\widehat{X}_{l} \in B\left(\overline{Y_{j}}\right)\right\}}\right) \mid M_{R} \leq 2 \delta m\right] & \leq 2 K_{2} \sum_{j=1}^{m} P\left(V_{j} \in \bar{R}_{Y} \mid M_{R} \leq 2 \delta m\right) \\
& \leq 4 K_{2} \delta m
\end{aligned}
$$

since $P\left(V_{j} \in \bar{R}_{Y} \mid M_{R} \leq 2 \delta m\right) \leq 1$ for $M_{R} \leq 2 \delta m$, and is zero for $M_{R}>2 \delta m$.

Recall from equations (5.10) and (5.12) that $\left|\Gamma\left(\mathcal{X}_{n}, \mathcal{Y}_{m}\right)-\Gamma\left(\widehat{\mathcal{X}}_{n}, \widehat{\mathcal{Y}}_{m}\right)\right|$ is bounded by

$$
\sum_{i=1}^{n} \mathbf{I}_{\left\{U_{i} \in \bar{R}_{X}\right\}}\left(N_{X}\left(B\left(X_{i}\right)\right)+N_{\widehat{X}}\left(B\left(\bar{X}_{i}\right)\right)\right)+\sum_{j=1}^{m} \mathbf{I}_{\left\{V_{j} \in \bar{R}_{Y}\right\}}\left(N_{\widehat{X}}\left(B\left(Y_{j}\right)\right)+N_{\widehat{X}}\left(B\left(\bar{Y}_{j}\right)\right)\right) .
$$

Since for positive real numbers $a, b, c$ with $a+b>c>\epsilon$ for some $\epsilon>0$ implies $a>\epsilon / 2$ or $b>\epsilon / 2$,

$$
\begin{aligned}
P\left(\frac{\left|\Gamma\left(\mathcal{X}_{n}, \mathcal{Y}_{m}\right)-\Gamma\left(\widehat{\mathcal{X}}_{n}, \widehat{\mathcal{Y}}_{m}\right)\right|}{n}>\epsilon\right) \leq & P\left(\sum_{i=1}^{n} \mathbf{I}_{\left\{U_{i} \in \bar{R}_{X}\right\}}\left(N_{X}\left(B\left(X_{i}\right)\right)+N_{\widehat{X}}\left(B\left(\bar{X}_{i}\right)\right)>\frac{n \epsilon}{2}\right)\right. \\
& +P\left(\sum_{j=1}^{m} \mathbf{I}_{\left\{V_{j} \in \bar{R}_{Y}\right\}}\left(N_{\widehat{X}}\left(B\left(Y_{j}\right)\right)+N_{\widehat{X}}\left(B\left(\overline{Y_{j}}\right)\right)\right)>\frac{n \epsilon}{2}\right)
\end{aligned}
$$




$$
\begin{aligned}
& \leq P\left(\sum_{i=1}^{n} \mathbf{I}_{\left\{U_{i} \in \bar{R}_{X}\right\}}\left(N_{X}\left(B\left(X_{i}\right)\right)+N_{\widehat{X}}\left(B\left(\bar{X}_{i}\right)\right)>\frac{n \epsilon}{2}\right)+P\left(M_{R}>2 \delta m\right)\right. \\
& +P\left(\sum_{j=1}^{m} \mathbf{I}_{\left\{V_{j} \in \bar{R}_{Y}\right\}}\left(N_{\widehat{X}}\left(B\left(Y_{j}\right)\right)+N_{\widehat{X}}\left(B\left(\overline{Y_{j}}\right)\right)\right)>\frac{n \epsilon}{2} \mid M_{R} \leq 2 \delta m\right) .
\end{aligned}
$$

Applying the Markov inequality to equations (5.11) and (5.15) yields that, for any $\epsilon>0$, when $n$ is sufficiently large,

$$
\begin{aligned}
P\left(\frac{\left|\Gamma\left(\mathcal{X}_{n}, \mathcal{Y}_{m}\right)-\Gamma\left(\widehat{\mathcal{X}}_{n}, \widehat{\mathcal{Y}}_{m}\right)\right|}{n}>\epsilon\right) & \leq \frac{\mathbf{E}\left[\sum_{i=1}^{n} \mathbf{I}_{\left\{U_{i} \in \bar{R}_{X}\right\}}\left(N_{X}\left(B\left(X_{i}\right)\right)+N_{\widehat{X}}\left(B\left(\bar{X}_{i}\right)\right)\right]\right.}{n \epsilon / 2}+P\left(M_{R}>2 \delta m\right) \\
& +\frac{\mathbf{E}\left[\sum_{j=1}^{m} \mathbf{I}_{\left\{V_{j} \in \bar{R}_{Y}\right\}}\left(N_{\widehat{X}}\left(B\left(Y_{j}\right)\right)+N_{\widehat{X}}\left(B\left(\overline{Y_{j}}\right)\right)\right) \mid M_{R} \leq 2 \delta m\right]}{n \epsilon / 2} \\
& \leq \frac{2 K_{1} \delta n}{n \epsilon}+\frac{4 K_{2} \delta m}{n \epsilon}+P\left(M_{R}>2 \delta m\right) \leq K \delta+P\left(M_{R}>2 \delta m\right)
\end{aligned}
$$

for some constant $K$ determined by $\epsilon$.

Recall that $M_{R}$ is a $\operatorname{Binomial}(m, \delta)$ random variable, by applying the Markov inequality, we obtain $P\left(M_{R}>\right.$ $2 m \delta)=P\left(M_{R}-m \delta>m \delta\right) \leq \frac{m \delta(1-\delta)}{(m \delta)^{2}}=\frac{(1-\delta)}{m \delta}$. Thus, for any fixed $\delta \in(0,1)$, when $m$ is sufficiently large (in particular, $\left.m>\frac{1-\delta}{\delta^{2}}\right)$, it follows that $P\left(M_{R}>2 \delta m\right) \leq \delta$. Hence, Inequality (5.16) reduces to

$$
P\left(\frac{\left|\Gamma\left(\mathcal{X}_{n}, \mathcal{Y}_{m}\right)-\Gamma\left(\widehat{\mathcal{X}}_{n}, \widehat{\mathcal{Y}}_{m}\right)\right|}{n}>\epsilon\right) \leq K \delta+\delta
$$

In the previous section, we proved that $\frac{\Gamma\left(\widehat{\mathcal{X}}_{n}, \widehat{\mathcal{Y}}_{m}\right)}{n} \rightarrow \mathcal{L}_{2}\left(r, \widehat{f}_{X}, \widehat{f}_{Y}\right)$ in probability as $n \rightarrow \infty$. Thus, when $n$ is sufficiently large, $P\left(\left|\frac{\Gamma\left(\widehat{\mathcal{X}}_{n}, \widehat{\mathcal{y}}_{m}\right)}{n}-\mathcal{L}_{2}\left(r, \widehat{f}_{X}, \widehat{f}_{Y}\right)\right|>\epsilon\right) \leq \delta$. Combining this inequality with Inequality (5.17) yields

$$
P\left(\left|\frac{\Gamma\left(\mathcal{X}_{n}, \mathcal{Y}_{m}\right)}{n}-\mathcal{L}_{2}\left(r, \widehat{f}_{X}, \widehat{f}_{Y}\right)\right|>2 \epsilon\right) \leq K \delta+2 \delta
$$

Corollary 4.2 says that $g_{2}(r)$ is bounded and continuous. Since $\hat{f}_{X} \rightarrow f_{X}$ and $\hat{f}_{Y} \rightarrow f_{Y}$ as $\delta \rightarrow 0(i . e$, as $k \rightarrow \infty)$, then by the dominated convergence theorem, it follows that as $\delta \rightarrow 0$

$$
\mathcal{L}_{2}\left(r, \widehat{f}_{X}, \widehat{f}_{Y}\right) \rightarrow \mathcal{L}_{2}\left(r, f_{X}, f_{Y}\right)
$$

Since $\delta>0$ can be arbitrarily small, we immediately obtain $\left.P\left(\mid \frac{\Gamma\left(\mathcal{X}_{n}, \mathcal{Y}_{m}\right)}{n}-\mathcal{L}_{2}\left(r, f_{X}, f_{Y}\right)\right] \mid>\epsilon\right) \rightarrow 0$ which finishes the proof of Theorem 1.8. 
Remark 5.4. The limiting function $\mathcal{L}_{2}\left(r, f_{X}, f_{Y}\right)$ gives the same value for uniform densities or when $f_{X}=f_{Y}$. However, since we have not proved whether $g_{2}$ is concave, we do not know yet if this limiting function achieves the maximum value when $f_{X}=f_{Y}$.

\section{EXTENSION TO HIGHER DIMENSIONS}

The extension of our results in $\mathbb{R}^{2}$ to higher dimensions $\mathbb{R}^{d}$ with $d>2$ can be achieved in a straightforward fashion with some modifications in the geometric arguments. The class cover problem (CCP) is already defined in any dissimilarity space (including $\mathbb{R}^{d}$ ) [3]. The LLNs for the domination number in $\mathbb{R}^{d}$ also follow from the ergodic theorem for multidimensional subadditive processes. One can prove the SLLN for the domination number of the CCCD generated by the Poisson points in $\mathbb{R}^{d}$, and then transfer the result to uniform data sets on $[0,1]^{d}$ with fixed sample sizes. The extension of constrained domination number induced by the $X$-points and $Y$-points with the covering balls bounded by the boundary of the study region is also straightforward.

In particular, in higher dimensions, $\mathbf{S}^{d}=\mathbb{R}_{+}^{d}$, the region $J_{q}$ becomes $J_{d, q}=[\mathbf{0}, q \mathbf{e})$, for $q>0$, and $\left|J_{d, q}\right|=q^{d}$. Furthermore, we assume that there are two independent Poisson processes $\left\{X_{i}\right\}$ and $\left\{Y_{j}\right\}$ in $\mathbb{R}^{d}$, with respective rates $\lambda_{X}$ and $\lambda_{Y}$. Then the extension of our main findings to higher dimensions are as below:

- Extension of Theorem 1.5: Let $\left\{X_{i}\right\}$ and $\left\{Y_{j}\right\}$ be two independent Poisson processes in $\mathbb{R}^{d}$, with rates $\lambda_{X}$ and $\lambda_{Y}$, respectively. If $\lambda_{Y} / \lambda_{X}=r, r \in(0, \infty)$, there exists a function $g_{d}$ such that $\lim _{t \rightarrow \infty, t \in \mathbb{R}} \frac{\bar{\Gamma}_{J_{d, t}}}{\left|J_{d, t}\right|}=$ $g_{d}(r)$ a.s.

- Extension of Theorem 1.6: Let $\left\{X_{i}\right\}$ and $\left\{Y_{j}\right\}$ be two independent Poisson processes in $\mathbb{R}^{d}$, with rates $\lambda_{X}$ and $\lambda_{Y}$, respectively If $\lambda_{Y} / \lambda_{X}=r, r \in(0, \infty)$, then $\lim _{t \rightarrow \infty, t \in \mathbb{R}} \frac{\Gamma_{J_{d, t}}}{\left|J_{d, t}\right|}=g_{d}(r)$ a.s., where $g_{d}(r)$ is as in the Extension of Theorem 1.5.

- Extension of Theorem 1.7: Let $X_{i} \stackrel{i i d}{\sim} U[0,1]^{d}$ for $i=1,2, \ldots, n$ and $Y_{j} \stackrel{i i d}{\sim} U[0,1]^{d}$ for $j=1,2, \ldots, m$ and $X_{i}$ and $Y_{j}$ be independent. If $m / n \rightarrow r, r \in(0, \infty)$, as $n \rightarrow \infty$, then $\lim _{n \rightarrow \infty} \frac{\Gamma_{n, m}}{n}=g_{d}(r)$ in probability, where $g_{d}(r)$ is as in the Extension of Theorem 1.5.

- Extension of Theorem 1.8: Let $X_{i}$ be i.i.d. from $f_{X}$ for $i=1,2, \ldots, n$ and $Y_{j}$ be i.i.d. from $f_{Y}$ for $j=1,2, \ldots, m$ and $X_{i}$ and $Y_{j}$ be independent. If the densities $f_{X}$ and $f_{Y}$ are positive, bounded, and continuous on their support, $[0,1]^{d}$, and $m / n \rightarrow r, r \in(0, \infty)$, as $n \rightarrow \infty$, then

$$
\lim _{n \rightarrow \infty} \frac{\Gamma_{n, m}}{n}=\mathcal{L}_{d}\left(r, f_{X}, f_{Y}\right) \text { in probability. }
$$

where $\mathcal{L}_{d}\left(r, f_{X}, f_{Y}\right)=\int \ldots \int g_{[0,1]^{d}} g_{d}\left(r \cdot \frac{f_{Y}(\mathbf{y})}{f_{X}(\mathbf{x})}\right) \cdot f_{X}(\mathbf{x}) \mathrm{d} \mathbf{x}$ where $\mathbf{x}=\left(x_{1}, \ldots, x_{d}\right), \mathbf{y}=\left(y_{1}, \ldots, y_{d}\right), d \mathbf{x}=$ $\mathrm{d} x_{1} \cdots \mathrm{d} x_{d}$ and $g_{d}(r)$ is as in the Extension of Theorem 1.5.

The proofs of these extensions are similar to those in the two-dimensional case. We only provide the proof of the higher dimensional version of Lemma 3.1 as an illustration.

Lemma 6.1. (Extension of Lem. 3.1) Let $J_{d, n}=[\mathbf{0}, n \mathbf{e})$ and $\bar{\Gamma}_{J_{d, n}}$ be the constrained domination number and $\Gamma_{J_{d, n}}$ be the unconstrained domination number. Then $\lim _{n \rightarrow \infty} \frac{\Gamma_{J_{d, n}}}{\left|J_{d, n}\right|}=\lim _{n \rightarrow \infty} \frac{\bar{\Gamma}_{J_{d, n}}}{\left|J_{d, n}\right|}$ a.s.

Proof. In $\mathbb{R}^{d}$, we have $J_{d, n}^{\prime}=\left[s_{n} \mathbf{e},\left(n-s_{n}\right) \mathbf{e}\right), J_{d, n}^{\prime \prime}=\left[2 s_{n} \mathbf{e},\left(n-2 s_{n}\right) \mathbf{e}\right)$, and $J_{d, n}^{\prime \prime \prime}=\left[(2+\sqrt{d}) s_{n} \mathbf{e},(n-\right.$ $\left.\left.(2+\sqrt{d}) s_{n}\right) \mathbf{e}\right)$. The quantity $s_{n}<\frac{n}{2(2+\sqrt{d})}$ will be chosen later. The $s_{n} \times s_{n}$ squares in $\mathbb{R}^{2}$ become $s_{n} \times$ $s_{n} \ldots \times s_{n}=s_{n}^{d}$ hypercubes between $J_{d, n}^{\prime}$ and $J_{d, n}^{\prime \prime}$. Let $F_{d, n}$ denote the event that all constrained covering balls of $X$-points in $J_{d, n}^{\prime \prime}$ are contained in $J_{d, n}$, and let $E_{d, n}$ denote the event that there exists at least one $Y$-point in each of the $s_{n}^{d}$ hypercubes between $J_{d, n}^{\prime}$ and $J_{d, n}^{\prime \prime}$. The probability of having at least one $Y$-point in one of these 
small hypercubes is $1-\exp \left(-s_{n}^{d} \lambda_{Y}\right)$, and the number of such small hypercubes is equal to $\frac{\left(n-s_{n}\right)^{d}-\left(n-4 s_{n}\right)^{d}}{s_{n}^{d}}$ which is less than equal to $2 d\left(n / s_{n}\right)^{d-1}$. Therefore, we have $P\left(E_{d, n}\right) \geq\left(1-\exp \left(-s_{n}^{d} \lambda_{Y}\right)\right)^{2 d\left(n / s_{n}\right)^{d-1}}$.

Next, we show that $E_{d, n} \subseteq F_{d, n}$, so it would follow that

$$
P\left(F_{d, n}\right) \geq\left(1-\exp \left(-s_{n}^{d} \lambda_{Y}\right)\right)^{2 d\left(n / s_{n}\right)^{d-1}}
$$

Suppose $Y_{j}$ is the $Y$-point closest to $X_{i}$, and let $Z_{i}$ be the closest point on $\partial\left(J_{d, n}\right)$, the boundary of $J_{d, n}$, to $X_{i}$, and let $P_{i}$ be the projection of $Y_{j}$ on the line segment joining $X_{i}$ and $Z_{i}$. Moreover, let $d\left(X_{i}, P_{i}\right)=b$ and $d\left(Y_{j}, P_{i}\right)=a$. Then the radius of the constrained covering hypersphere $\bar{B}_{J_{d, n}}\left(X_{i}\right)$ is $\sqrt{a^{2}+b^{2}}$, Since $a \leq s_{n}$, we have $\sqrt{a^{2}+b^{2}} \leq \sqrt{s_{n}^{2}+b^{2}} \leq s_{n}+b$. But the distance from $X_{i}$ to $\partial\left(J_{d, n}\right)$ is greater or equal than $s_{n}+b$, so we obtain $\bar{B}_{J_{d, n}}\left(X_{i}\right)$ is contained in $J_{d, n}$.

Now let $\Delta_{J_{d, n}}=\bar{\Gamma}_{J_{d, n}}-\Gamma_{J_{d, n}}$. As in the two-dimensional case, $\Delta_{J_{d, n}} \geq 0$. Given the event $F_{d, n}$, the constrained covering hypersphere resizing can only occur for those $X$-points in $J_{d, n} \backslash J_{d, n}^{\prime \prime}$ and the resized hyperspheres do not intersect $J_{d, n}^{\prime \prime \prime}$. The reason is that there is a closest $Y_{j}$ to $X_{i}$ in the hypercube and that the radius of the resized covering hypersphere $B\left(X_{i}\right)$ is $\sqrt{b_{1}^{2}+b_{2}^{2}+\cdots+b_{d}^{2}}$. Since $b_{i} \leq s_{n}$ for all $i=1,2, \ldots, d$ we have $\sqrt{b_{1}^{2}+b_{2}^{2}+\cdots+b_{d}^{2}} \leq \sqrt{d} s_{n}$ and the distance from $X_{i}$ to the boundary of $J_{d, n}^{\prime \prime \prime}$ is greater or equal to $\sqrt{d} s_{n}$. Therefore, $\Delta_{J_{d, n}} \leq N_{X}\left(J_{d, n} \backslash J_{d, n}^{\prime \prime \prime}\right)$.

By the arguments in the preceding paragraph

$$
P\left(\frac{\Delta_{J_{d, n}}}{\left|J_{d, n}\right|}>\epsilon\right) \leq P\left(N_{X}\left(J_{d, n} \backslash J_{d, n}^{\prime \prime \prime}\right)>\epsilon\left|J_{d, n}\right|\right)+P\left(F_{d, n}^{c}\right)
$$

and by the union bound (for the middle inequality below), for $n$ sufficiently large and $s_{n}=\sqrt[d]{(d+\delta) \log (n) / \lambda_{Y}}$ for some $\delta \in(0,1)$

$$
P\left(F_{d, n}^{c}\right) \leq P\left(E_{d, n}^{c}\right) \leq \frac{2 d n^{d-1}}{s_{n}^{d-1}} e^{-s_{n}^{d} \lambda_{Y}} \leq n^{d-1} e^{-(d+\delta) \log n}
$$

which is summable $n$, but also $\mathbf{E}\left[N_{X}\left(J_{d, n} \backslash J_{d, n}^{\prime \prime \prime}\right)\right] \sim c^{\prime} n^{d-1} s_{n}$, so by a Chernoff bound for the Binomial distribution, for $n$ sufficiently large,

$$
P\left(N_{X}\left(J_{d, n} \backslash J_{d, n}^{\prime \prime \prime}\right)>\epsilon n^{d /(d-1)}\right) \leq \exp \left(-n^{d-1}\right)
$$

which is also summable in $n$.

Notice that this choice of $s_{n}$ implies $P\left(F_{d, n}^{c}\right) \rightarrow 0$ as $n \rightarrow \infty$. By the Borel-Cantelli Lemma, the above calculations imply that $\frac{\Delta_{J_{d, n}}}{\left|J_{d, n}\right|} \stackrel{a . s}{\longrightarrow} 0$. Therefore

$$
\lim _{n \rightarrow \infty} \frac{\Gamma_{J_{d, n}}}{\left|J_{d, n}\right|}=\lim _{n \rightarrow \infty} \frac{\bar{\Gamma}_{J_{d, n}}}{\left|J_{d, n}\right|} \text { a.s. }
$$

which completes the proof.

The proofs of the high dimensional extension of Theorems 1.6 and 1.7 also follow with similar geometric adjustments above for higher dimensions. As main changes in proving the extension of Lemma 4.1, we modify the cases of adding and deleting $Y$-points as follows. In the case of adding one new $Y$-point: $Y_{a}$, we equally divide the hypercube centered at $Y_{a}$ with side length $4 b$ into $8^{d}$ smaller hypercubes, and refer to the $8^{d}$ small hyperspheres inscribed in the hypercubes with radius $b / 4$ as grid hyperspheres. As the Poisson process $Y$ has 
rate $\lambda_{Y}$, the probability that a particular grid hypersphere covers no $Y$-point is $\exp \left(-\frac{\pi^{d / 2}(b / 4)^{d} \lambda_{Y}}{\Gamma(d / 2+1)}\right)$ where $\Gamma(x)=\int_{0}^{\infty} t^{x-1} e^{-t} d t$ is the usual gamma function. Then the proof proceeds as in the $d=2$ case with inserting this probability appropriately. The same conclusion of Corollary 4.2 also holds for $g_{d}(r)$ with the proof requiring almost no adjustment for extending to higher dimensions.

The extension of Theorem 1.8 can be proved similarly to the one in the two dimensional setting: We first generalize extension of Theorem 1.7 to piece-wise constant densities, then extend it to the continuous case. The proofs are analogous to those in the two dimensional setting.

Remark 6.2. Notice that $g_{2}(r)$ in Theorems 1.5-1.8 (and likewise for $g_{d}(r)$ in Sect. 6) is not explicitly available in contrast to the one-dimensional case. In applications, this might constitute a drawback which can be overcome by estimating $g_{2}(r)$ empirically by Monte Carlo simulations (which is not pursued in this article).

\section{DisCUSSION AND CONCLUSIONS}

We study the class cover problem (CCP) of random point sets in two (or higher) dimensions. In particular, given two classes $\mathcal{X}$ and $\mathcal{Y}$ in a sample space $\Omega$ with corresponding random variables $X_{i}$ and $Y_{j}$, respectively, the covering ball of $X_{i}$, denoted by $B\left(X_{i}, r_{i}\right)$, is the set of points $\omega$ in $\Omega$ such that $X_{i}$ is closer to $\omega$ than to any other $Y_{j}$. That is, letting $\mathcal{X}_{n}=\left\{X_{1}, X_{2}, \ldots, X_{n}\right\}$ and $\mathcal{Y}_{m}=\left\{Y_{1}, Y_{2}, \ldots, Y_{m}\right\}, B\left(X_{i}, r_{i}\right)$ is the ball with radius $r_{i} \leq \min _{j} d\left(X_{i}, Y_{j}\right)$. The goal in the CCP is to minimize the number of covering balls needed to cover all $X$-points, $\mathcal{X}_{n}$. This goal is equivalent to finding a minimum dominating set for the digraph called the class cover catch digraph (CCCD). A CCCD has vertex set $\mathcal{X}_{n}$ and an arc (i.e., directed edge) from $X_{i}$ to $X_{j}$ is inserted if $X_{j} \in B\left(X_{i}, r_{i}\right)$.

The CCP (and hence the CCCD) is motivated by its application in pattern classification. DeVinney and Wierman proved the Strong Law of Large Numbers (SLLN) for the uniform distribution in one dimension [10], and Wierman and Xiang extended the SLLN to the case of general distributions in one dimension [31]. We study the behavior of the domination number of CCCDs when $X$-and $Y$-points are coming from independent Poisson point processes in $\mathbb{R}^{2}$, as well as $\mathcal{X}_{n}$ and $\mathcal{Y}_{m}$ are chosen uniformly from the unit square and also $X$ - and $Y$-points have positive, bounded and continuous densities. In the Poisson process case, we prove a SLLN result for the domination number; i.e., we show that the domination number (properly scaled) converges almost surely to a limiting function, denoted $g_{d}(r)$, where $r$ is the ratio of the rate of the Poisson process for $Y$ points to that for $X$ points in $\mathbb{R}^{d}$. The proof proceeds by applying a result on almost sure convergence of subadditive processes for a constrained domination number, and then showing that the difference between the unconstrained and constrained domination number vanishes in the limit, which is shown by extensive geometric and probabilistic computations. For the case of uniformly distributed points in the unit square, we obtain a WLLN result (i.e., convergence in probability) for the scaled domination number. Finally, we generalize this result to the case where the densities are positive, bounded and continuous on $[0,1]^{2}$ and then extend the results to higher dimensions.

The solutions to the CCP (i.e., the minimum dominating sets of the CCCDs) are employed to build classifiers. For example, the balls around the members of the minimum dominating sets of the CCCDs can be used to construct discriminant regions for assigning class labels (see [8] for more detail). CCCD-based methods have been shown to have relatively good performance in classification (see $[9,23])$ and also to be robust to the class imbalance problem [20].

One major drawback of CCCDs is that the (exact or asymptotic) distribution of its domination number is only available in the one dimensional case. Despite this difficulty, we were able to show SLLN and WLLN results for the domination number in the Poisson process and uniform distribution cases, respectively, and also determine some properties of the limit of the domination number. The difficulties in extending the nice properties and results in the one-dimensional case to higher dimensions are discussed in [5]. These difficulties mainly arise from the lack of a natural ordering of points in two or higher dimensions, and when $\mathcal{Y}_{m}$ partitions the space into cells, the balls are not necessarily restricted to the particular cell their center reside in. CCCDs were generalized to proximity catch digraphs (PCDs) in [4] where distribution of the domination number of PCDs is more tractable than that of CCCDs. The current work also suggests that domination number of CCCDs 
might have asymptotic normality, so a CLT result is also a topic of prospective research. Moreover, this prospect is also highly contingent on finding the explicit forms of the limiting function, $g_{d}(r)$, at least for $d=2$, which is also an open problem.

\section{Appendix A.}

\section{A.1 Proof of Lemma 4.1}

For any $\delta>0$ and $\epsilon>0$, by the law of total probability, we have

$$
\begin{aligned}
& P\left(\frac{\left|\Delta_{n, M_{n}}\right|}{n}>\epsilon\right)=\sum_{\rho_{n}>\delta n} P\left(\frac{\left|\Delta_{n, M_{n}}\right|}{n}>\epsilon|| m-M_{n} \mid=\rho_{n}\right) P\left(\left|m-M_{n}\right|=\rho_{n}\right) \\
& +\sum_{1 \leq \rho_{n} \leq \delta n} P\left(\frac{\left|\Delta_{n, M_{n}}\right|}{n}>\epsilon \mid M_{n}-m=\rho_{n}\right) P\left(M_{n}-m=\rho_{n}\right)+P\left(\frac{\left|\Delta_{n, M_{n}}\right|}{n}>\epsilon \mid M_{n}-m=0\right) P\left(M_{n}-m=0\right) \\
& +\sum_{1 \leq \rho_{n} \leq \delta n} P\left(\frac{\left|\Delta_{n, M_{n}}\right|}{n}>\epsilon \mid m-M_{n}=\rho_{n}\right) P\left(m-M_{n}=\rho_{n}\right) .
\end{aligned}
$$

Observe that the first term in the right hand side is for the case of $m$ and $M_{n}$ differing by more than $\delta n$ from each other, the second term is for deleting $\rho_{n} Y$-points, the third term is when there is no need of change in the number of $Y$-points, and finally the last term is for adding $\rho_{n} Y$-points. Observe that if $m=M_{n}$, then $\Delta_{n, M_{n}}=0$, thus $P\left(\frac{\left|\Delta_{n, M_{n}}\right|}{n}>\epsilon \mid M_{n}-m=0\right)=0$, hence the third term above vanishes. Since $P\left(\frac{\left|\Delta_{n, M_{n}}\right|}{n}>\epsilon|| m-M_{n} \mid=\rho_{n}\right) \leq 1$ and $\sum_{\rho_{n}>\delta n} P\left(\left|m-M_{n}\right|=\rho_{n}\right)=P\left(\left|m-M_{n}\right|>\delta n\right)$ the probability above can be bounded as follows:

$$
\begin{aligned}
P\left(\frac{\left|\Delta_{n, M_{n}}\right|}{n}>\epsilon\right) \leq & P\left(\left|M_{n}-m\right|>\delta n\right)+\sum_{1 \leq \rho_{n} \leq \delta n} P\left(\frac{\left|\Delta_{n, M_{n}}\right|}{n}>\epsilon \mid M_{n}-m=\rho_{n}\right) P\left(M_{n}-m=\rho_{n}\right) \\
& +\sum_{1 \leq \rho_{n} \leq \delta n} P\left(\frac{\left|\Delta_{n, M_{n}}\right|}{n}>\epsilon \mid m-M_{n}=\rho_{n}\right) P\left(m-M_{n}=\rho_{n}\right)
\end{aligned}
$$

We bound the first term in the right hand side of equation (A.1) as follows. Since $m / n \rightarrow r$, when $n$ is sufficiently large, we have

$$
\frac{|m-r n|}{n}<\delta / 2
$$

thus, by triangle inequality and using equation (A.2)

$$
\begin{aligned}
P\left(\left|M_{n}-m\right|>\delta n\right)=P\left(\frac{\left|M_{n}-m\right|}{n}>\delta\right) & \leq P\left(\frac{\left|M_{n}-r n\right|}{n}+\frac{|m-r n|}{n}>\delta\right) \\
& \leq P\left(\frac{\left|M_{n}-r n\right|}{n} \geq \frac{\delta}{2}\right)=P\left(\left|M_{n}-r n\right| \geq \delta n / 2\right) .
\end{aligned}
$$

Applying a similar argument as in page 432 of [10], which uses Chernoff's Theorem, the above is further bounded as follows:

$$
P\left(\left|M_{n}-m\right|>\delta n\right) \leq P\left(\left|M_{n}-r n\right| \geq \frac{\delta n}{2}\right) \leq K e^{-k \delta n}
$$




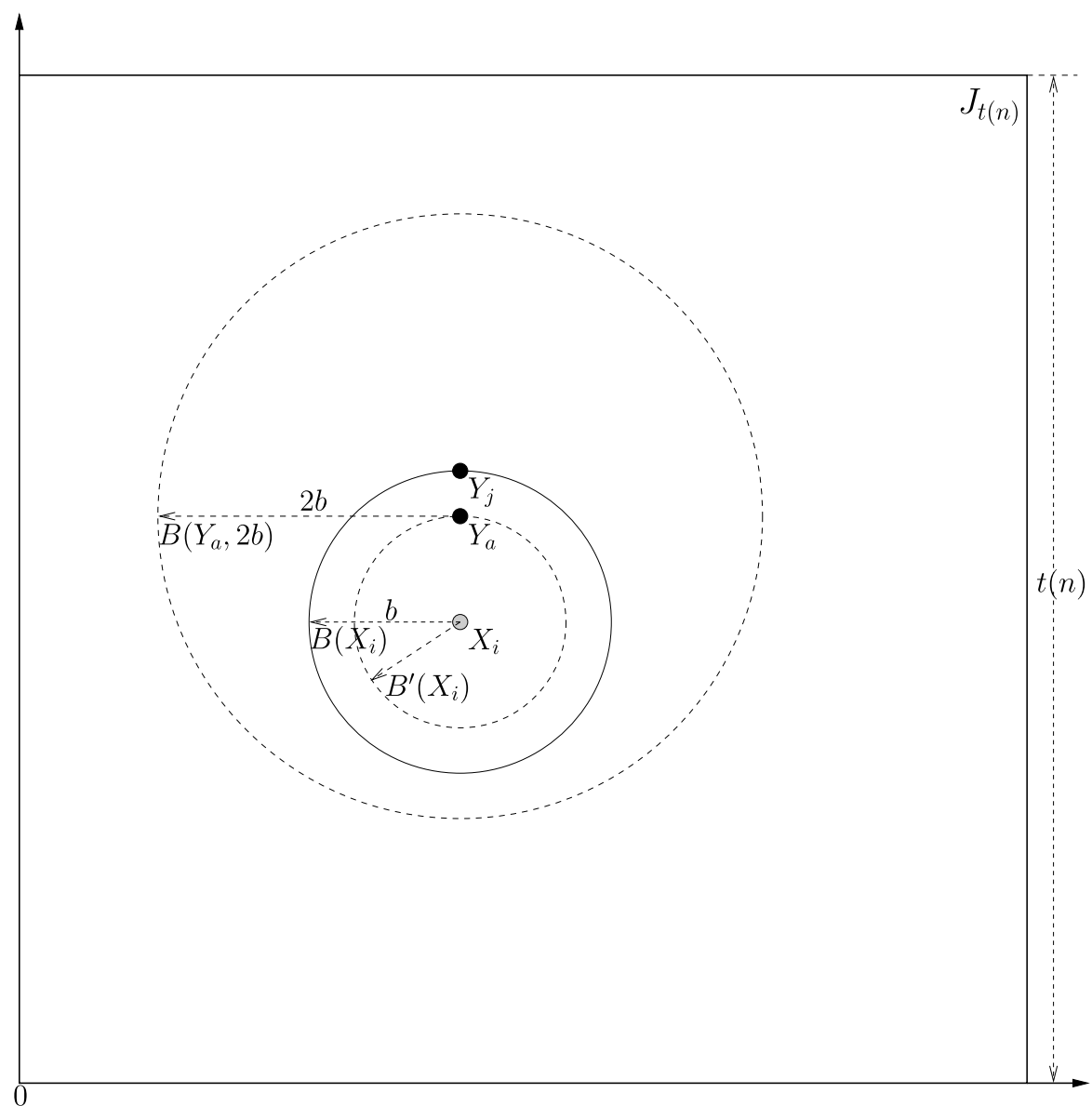

Figure A.1. The result of adding one new point $Y_{a}$.

for some constants $K, k>0$ and sufficiently large $n$. Next, we bound the second and third terms in (A.1) by considering the cases of adding and deleting up to $\delta n Y$-points.

Case 1: Adding up to $\delta n Y$-Points. We first consider the case of adding one new $Y$-point: $Y_{a}$, i.e., $m-M_{n}=1$.

As illustrated in Figure A.1, if $Y_{a}$ falls into the covering ball $B\left(X_{i}\right)$ of some point $X_{i}$, the covering ball $B\left(X_{i}\right)$ will decrease to $B^{\prime}\left(X_{i}\right)$ so that the domination number may increase (but never decreases). Such an increase can be at most the number of $X$-points in $B\left(X_{i}\right)$.

Note that it is possible for $Y_{a}$ to fall into more than one covering ball. To take this into account, define the random variable $B_{a}$ as the maximum radius of all balls that contain $Y_{a}$ but contain no other $Y$-points. We know that given $B_{a}=b>0$, the covering balls into which $Y_{a}$ could fall must be contained in the ball $B\left(Y_{a}, 2 b\right)$, which is the ball centered at $Y_{a}$ with radius $2 b$. Otherwise, if there exists a covering ball that contains $Y_{a}$ but is not contained in $B\left(Y_{a}, 2 b\right)$, then that covering ball must have a radius greater than $b$ but contains no $Y$-points, which contradicts $B_{a}=b$. Therefore, $\Delta_{n, M_{n}}$ is bounded above by the number of $X$-points in $B\left(Y_{a}, 2 b\right)$, thus

$$
0 \leq \Delta_{n, M_{n}} \leq N_{X}\left(B\left(Y_{a}, 2 b\right)\right)=\sum_{i=1}^{n} \mathbf{I}_{\left\{X_{i} \in B\left(Y_{a}, 2 b\right)\right\}}
$$




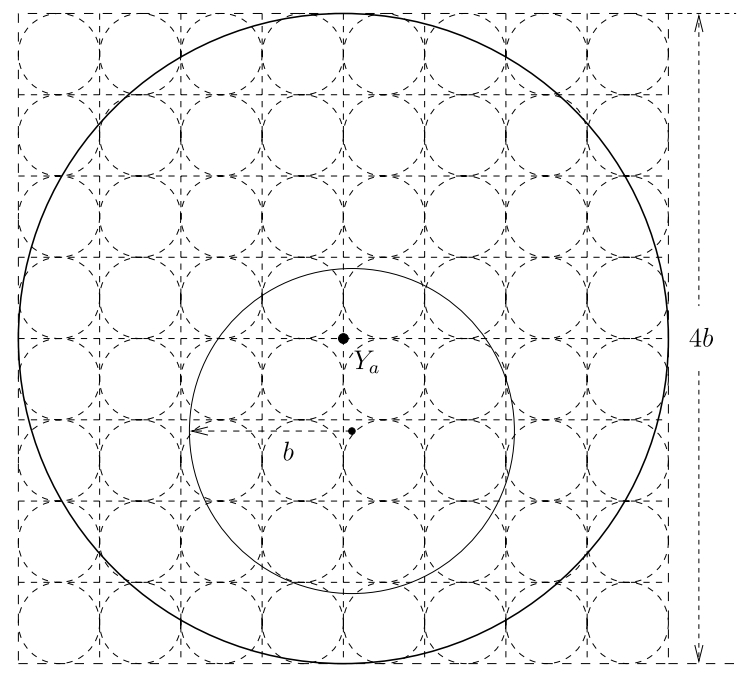

FiguRE A.2. The affected region of an added point $Y_{a}$.

Next, we calculate an upper bound for $P\left(B_{a}>b\right)$. Define $E\left(Y_{a}, b\right)$ to be the event that "there exists a ball in $B\left(Y_{a}, 2 b\right)$ with radius $b$ which contains no $Y$-point." Note that in the definition above, the ball is a subset of $B\left(Y_{a}, 2 b\right)$, but is not necessarily centered at an $X$-point or a $Y$-point. From the definition of $B_{a}$, we have $\left\{B_{a}>b\right\} \subset E\left(Y_{a}, b\right)$.

Thus, we will find an upper bound for $P\left(E\left(Y_{a}, b\right)\right)$. As shown in Figure A.2, suppose we equally divide the square centered at $Y_{a}$ - with sides parallel to the coordinate axes - with side length $4 b$ into $8^{2}=64$ smaller squares, and refer to the 64 small balls inscribed in the squares with radius $b / 4$ as grid balls. If $E\left(Y_{a}, b\right)$ occurs, i.e., there exists a ball in $B\left(Y_{a}, 2 b\right)$ with radius $b$ which contains no $Y$-points, then that ball must contain a grid ball that covers no $Y$-point (as illustrated in Fig. A.2).

Therefore, if $E\left(Y_{a}, b\right)$ occurs, there must be a grid ball containing no $Y$-point. Since the Poisson process $Y$ has rate $\lambda_{Y}$, we know the probability that a particular grid ball covers no $Y$-point is $\exp \left(-\pi(b / 4)^{2} \lambda_{Y}\right)$. Since $\lambda_{Y}=r$, and by Boole's inequality, $P\left(B_{a}>b\right) \leq P\left(E\left(Y_{a}, b\right)\right) \leq 64 \exp \left(-\pi r(b / 4)^{2}\right)$. Applying (A.4), we have

$$
P\left(\frac{\left|\Delta_{n, M_{n}}\right|}{n}>\epsilon \mid m-M_{n}=1, B_{a}=b\right) \leq P\left(N_{X}\left(B\left(Y_{a}, 2 b\right)\right)>n \epsilon \mid m-M_{n}=1, B_{a}=b\right) .
$$

Since the $X$-points are independent of the $Y$-points, all $X_{i}$ are identically distributed, and $B_{a}$ is defined independently of the $X$-points, the right side of this inequality equals the unconditional probability. Then, by Markov's Inequality, we have

$$
P\left(N_{X}\left(B\left(Y_{a}, 2 b\right)\right)>n \epsilon\right) \leq \frac{\mathbf{E}\left[\sum_{i=1}^{n} \mathbf{I}_{\left.\left\{X_{i} \in B\left(Y_{a}, 2 b\right)\right)\right\}}\right]}{n \epsilon}=\frac{P\left(X_{1} \in B\left(Y_{a}, 2 b\right)\right)}{\epsilon} .
$$

Note that if $B\left(Y_{a}, 2 b\right)$ is contained in $J_{T(n)}$, then $P\left(X_{1} \in B\left(Y_{a}, 2 b\right)\right)=\pi(2 b)^{2} /\left|J_{T(n)}\right|$. However, if $Y_{a}$ is near the boundary of $J_{T(n)}$, then it is possible that only part of $B\left(Y_{a}, 2 b\right)$ is contained in $J_{T(n)}$, so $P\left(X_{1} \in B\left(Y_{a}, 2 b\right)\right) \leq$ $\pi(2 b)^{2} /\left|J_{T(n)}\right|$. Summarizing the discussion above, we obtain

$$
P\left(\frac{\left|\Delta_{n, M_{n}}\right|}{n}>\epsilon \mid m-M_{n}=1, B_{a}=b\right) \leq \frac{\pi(2 b)^{2}}{\left|J_{T(n)}\right| \cdot \epsilon} .
$$


Hence, given $T(n)$,

$$
\begin{aligned}
& P\left(\frac{\left|\Delta_{n, M_{n}}\right|}{n}>\epsilon \mid m-M_{n}=1\right)=\int_{b=0}^{\sqrt{2} T(n)} P\left(\frac{\left|\Delta_{n, M_{n}}\right|}{n}>\epsilon \mid m-M_{n}=1, B_{a}=b\right) \mathrm{d} F_{B_{a}}(b) \\
& \leq \int_{b=0}^{\sqrt{2} T(n)} \frac{\pi(2 b)^{2}}{\left|J_{T(n)}\right| \cdot \epsilon} \mathrm{d}\left(1-P\left(B_{a}>b\right)\right) \leq \int_{b=0}^{\sqrt{2} T(n)} P\left(B_{a}>b\right) \mathrm{d}\left(\frac{\pi(2 b)^{2}}{\left|J_{T(n)}\right| \cdot \epsilon}\right) .
\end{aligned}
$$

since $d\left(1-P\left(B_{a}>b\right)\right)=-d\left(P\left(B_{a}>b\right)\right)$ and $P\left(B_{a}>\sqrt{2} T(n)\right)=0$. Recalling that $P\left(B_{a}>b\right) \leq$ $64 \exp \left(-\pi r(b / 4)^{2}\right)$, we further bound the above as follows:

$$
P\left(\frac{\left|\Delta_{n, M_{n}}\right|}{n}>\epsilon \mid m-M_{n}=1\right) \leq \int_{b=0}^{\sqrt{2} T(n)} 64 \exp \left(-\pi r(b / 4)^{2}\right) \mathrm{d}\left(\frac{\pi(2 b)^{2}}{\left|J_{T(n)}\right| \cdot \epsilon}\right) \leq \frac{C}{\left|J_{T(n)}\right|},
$$

where $C>0$ is a constant. Therefore, without conditioning on $T(n)$, we have

$$
P\left(\frac{\left|\Delta_{n, M_{n}}\right|}{n}>\epsilon \mid m-M_{n}=1\right) \leq C \cdot \mathbf{E}\left[\frac{1}{\left|J_{T(n)}\right|}\right] .
$$

Next, we consider the case of adding one or more new $Y$-points: $Y_{a}^{1}, \ldots, Y_{a}^{m-M_{n}}$, when $m-M_{n}>1$. Similarly define $B_{a}^{l}$ as the radius of the covering balls containing $Y_{a}^{l}, l=1, \ldots, m-M_{n}$. Given $m-M_{n}=\rho_{n} \in$ $\{1,2, \ldots, \delta n\}$ and $B_{a}^{l}=b_{l}>0$, by applying the same arguments to obtain (A.5), we have

$$
P\left(\frac{\left|\Delta_{n, M_{n}}\right|}{n}>\epsilon \mid m-M_{n}=\rho_{n}, B_{a}^{l}=b_{l}\right) \leq \frac{P\left(X_{i} \in \cup_{l=1}^{\rho_{n}} B\left(Y_{a}^{l}, 2 b_{l}\right)\right)}{\epsilon} \leq \sum_{l=1}^{\rho_{n}} \frac{\pi\left(2 b_{l}\right)^{2}}{\left|J_{T(n)}\right| \cdot \epsilon} .
$$

Using $P\left(B_{a}^{l}>b_{l}\right) \leq 64 \exp \left(-\pi r\left(b_{l} / 4\right)^{2}\right)$ for each $l$ as before, and recalling we have chosen $\rho_{n} \leq \delta n$, we can finally get the following bound:

$$
P\left(\frac{\left|\Delta_{n, M_{n}}\right|}{n}>\epsilon \mid m-M_{n}=\rho_{n}\right) \leq \rho_{n} \cdot C \cdot \mathbf{E}\left[\frac{1}{\left|J_{T(n)}\right|}\right] \leq \delta n \cdot C \cdot \mathbf{E}\left[\frac{1}{\left|J_{T(n)}\right|}\right] .
$$

Hence,

$$
\begin{aligned}
& \sum_{1 \leq \rho_{n} \leq \delta n} P\left(\frac{\left|\Delta_{n, M_{n}}\right|}{n}>\epsilon \mid m-M_{n}=\rho_{n}\right) P\left(m-M_{n}=\rho_{n}\right) \leq \sum_{1 \leq \rho_{n} \leq \delta n} \delta n \cdot C \cdot \mathbf{E}\left[\frac{1}{\left|J_{T(n)}\right|}\right] P\left(m-M_{n}=\rho_{n}\right) \\
= & \delta n \cdot C \cdot \mathbf{E}\left[\frac{1}{\left|J_{T(n)}\right|}\right] \sum_{1 \leq \rho_{n} \leq \delta n} P\left(m-M_{n}=\rho_{n}\right) \quad\left(\text { since summation equals } P\left(1 \leq m-M_{n} \leq \delta n\right)\right) \\
\leq & \delta n \cdot C \cdot \mathbf{E}\left[\frac{1}{\left|J_{T(n)}\right|}\right] \quad\left(\text { since } P\left(1 \leq m-M_{n} \leq \delta n\right) \leq 1\right) \\
\leq & \delta C_{1} \text { (since } \mathbf{E}\left[\frac{n}{\left|J_{T(n)}\right|}\right] \text { is bounded). }
\end{aligned}
$$

for some constant $C_{1}>0$.

Case 2: Deleting up to $\delta n Y$-Points. In contrast to adding a point, deleting an existing point, $Y_{d}$, can only decrease the domination number or leave it unchanged. 


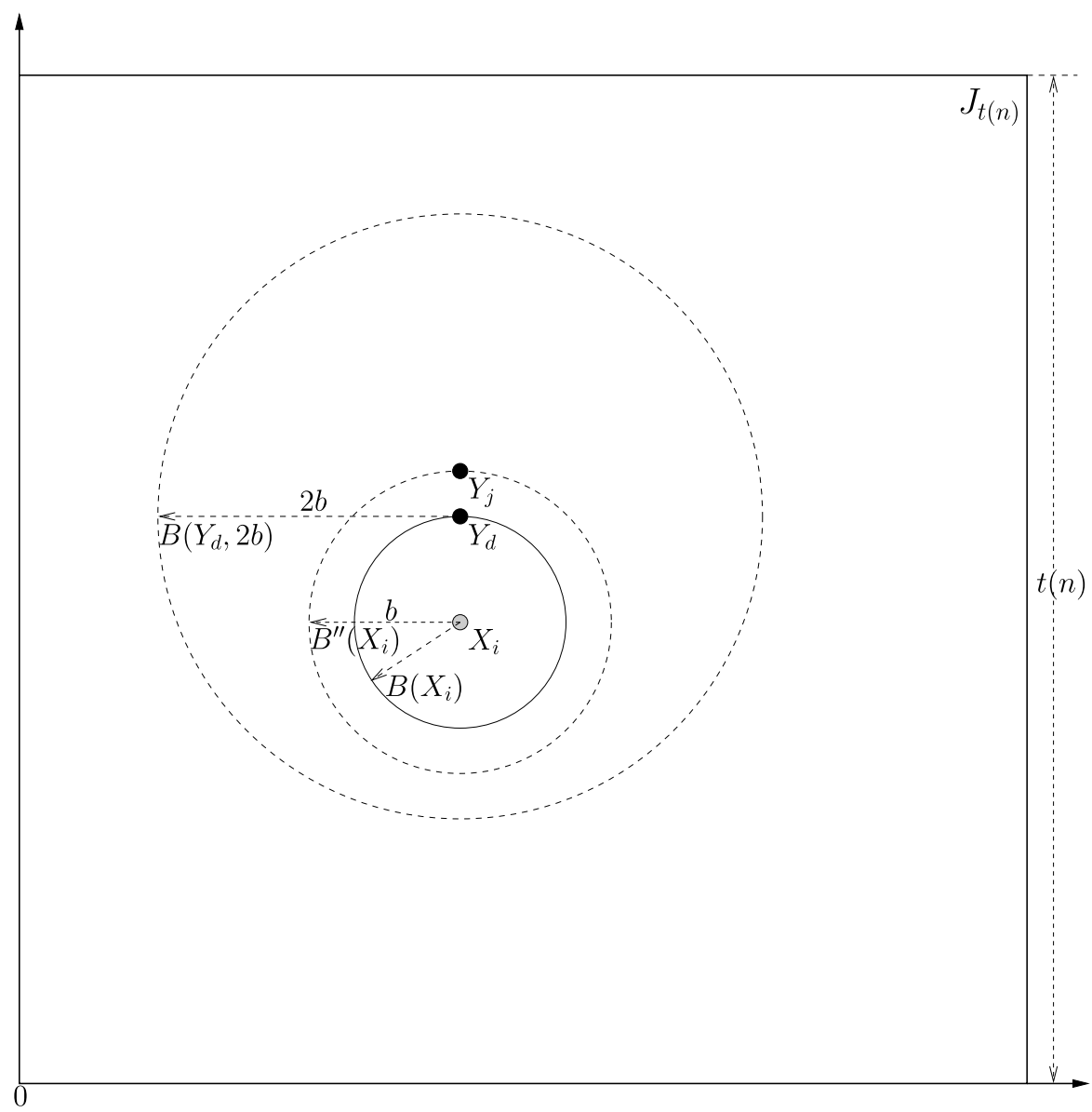

Figure A.3. The result of deleting one existing point $Y_{d}$.

As illustrated in Figure A.3, if $Y_{d}$ is on the boundary of $B\left(X_{i}\right)$ of some $X_{i}$, then deleting $Y_{d}$ will cause $B\left(X_{i}\right)$ to increase to $B^{\prime \prime}\left(X_{i}\right)$, which we refer to as the enlarged covering ball. The enlarged covering ball $B^{\prime \prime}\left(X_{i}\right)$ has a radius equal to the distance between $X_{i}$ and the second nearest $Y$-point, $Y_{j}$. It is worth noting that the domination number can decrease by at most the number of $X$-points in $B^{\prime \prime}\left(X_{i}\right) \backslash B\left(X_{i}\right)$.

It is also possible for $Y_{d}$ to fall into more than one enlarged covering ball. Refer to the original $Y$-points except $Y_{d}$ as $Y^{\prime}$-points. Define the random variable $B_{d}$ as the maximum radius of all balls that contain $Y_{d}$ but contain no $Y^{\prime}$-points. Given $B_{d}=b>0$, the enlarged covering balls into which $Y_{d}$ could fall must be contained in the ball $B\left(Y_{d}, 2 b\right)$. Otherwise, if there exists an enlarged covering ball that contains $Y_{d}$ but is not contained in $B\left(Y_{d}, 2 b\right)$, then that enlarged covering ball must have a radius greater than $b$ but contain no $Y^{\prime}$-point, which contradicts $B_{d}=b$. Therefore, $\left|\Delta_{n, M_{n}}\right|$ is bounded above by the number of $X$-points in $B\left(Y_{d}, 2 b\right)$, thus

$$
-\sum_{i=1}^{n} \mathbf{I}_{\left\{X_{i} \in B\left(Y_{d}, 2 b\right)\right\}} \leq \Delta_{n, M_{n}} \leq 0 .
$$

Define the event $E^{\prime}\left(Y_{d}, b\right)=\left\{\exists\right.$ a ball in $B\left(Y_{d}, 2 b\right)$ with radius $b$ which contains no $Y^{\prime}$-point $\}$. As in the case of adding one point, it is easy to see that $\left\{B_{d}>b\right\} \subset E^{\prime}\left(Y_{d}, b\right)$. Hence, conditioning on $T(n)$, we can get the 
following upper bound for $P\left(B_{d}>b\right)$ :

$$
P\left(B_{d}>b\right) \leq P\left(E^{\prime}\left(Y_{d}, b\right)\right) \leq 64 \times P\left(\text { a grid ball contains no } Y^{\prime} \text {-point }\right)=64\left(1-\frac{\pi(b / 4)^{2}}{T(n)^{2}}\right)^{m} .
$$

Therefore, using the same argument as in the case of adding points, for any $\rho_{n} \in\{1,2, \ldots, \delta n\}$, we obtain

$$
\begin{aligned}
P\left(\frac{\left|\Delta_{n, M_{n}}\right|}{n}>\epsilon \mid M_{n}-m=\rho_{n}\right) & \leq \delta n \cdot E\left[\int_{b=0}^{\sqrt{2} T(n)} 64\left(1-\frac{\pi(b / 4)^{2}}{T(n)^{2}}\right)^{m} \mathrm{~d}\left(\frac{\pi(2 b)^{2}}{T(n)^{2} \cdot \epsilon}\right)\right] \\
& \leq \delta n \frac{C^{\prime}}{m} \leq \delta C_{2}
\end{aligned}
$$

for some constant $C_{2}>0$. Therefore,

$$
\begin{aligned}
\sum_{1 \leq \rho_{n} \leq \delta n} P\left(\frac{\left|\Delta_{n, M_{n}}\right|}{n}>\epsilon \mid M_{n}-m=\rho_{n}\right) P\left(M_{n}-m=\rho_{n}\right) & \leq \delta C_{2} \sum_{1 \leq \rho_{n} \leq \delta n} P\left(M_{n}-m=\rho_{n}\right) \\
& =\delta C_{2} P\left(1 \leq M_{n}-m \leq \delta n\right) \leq \delta C_{2} .
\end{aligned}
$$

Substituting the bounds found in equations (A.3), (A.6), and (A.7) into Inequality (A.1), it follows that

$$
P\left(\frac{\left|\Delta_{n, M_{n}}\right|}{n}>\epsilon\right) \leq K e^{-k \delta n}+\left(C_{1}+C_{2}\right) \delta
$$

For any fixed $\delta>0$, the first term $K e^{-k \delta n}$ goes to 0 as $n \rightarrow \infty$. Also, since $\delta>0$ can be arbitrarily small, we conclude that $P\left(\frac{\left|\Delta_{n, M_{n}}\right|}{n}>\epsilon\right) \rightarrow 0$, so $\frac{\Delta_{n, M_{n}}}{n} \rightarrow 0$ in probability.

Remark A.1. Notice that when adding points, $P\left(B_{a}>b\right)$ has an exponentially decaying bound, while when deleting points, $P\left(B_{d}>b\right)$ has a polynomially decaying bound. The main difference between these two cases is that when adding points, we add from a Poisson process with rate $\lambda_{Y}$, while deleting points we delete from a uniform distribution in the region of interest.

\section{A.2 Proof of Corollary 4.2}

$-g_{2}(r)$ is bounded: To prove this, we show that $g_{2}(r) \in[0,1]$. For integer $n$, we showed that $\lim _{n \rightarrow \infty} \frac{\bar{\Gamma}_{J_{n}}}{\left|J_{n}\right|}=$ $g_{2}(r)$ a.s., where $\lambda_{X}=1$ and $\lambda_{Y}=r$ are assumed.

Since $0 \leq \frac{\bar{\Gamma}_{J_{n}}}{\left|J_{n}\right|} \leq \frac{N_{X}\left(J_{n}\right)}{\left|J_{n}\right|}$ and $\frac{N_{X}\left(J_{n}\right)}{\left|J_{n}\right|} \stackrel{a . s}{\longrightarrow} \lambda_{X}=1$, it follows that $0 \leq g_{2}(r)=\lim _{n \rightarrow \infty} \frac{\bar{\Gamma}_{J_{n}}}{\left|J_{n}\right|} \leq 1$.

- $g_{2}(r)$ is an increasing function of $r$ : Next, we show that $g_{2}(r)$ increases as $r$ increases. We first suppose that, for any $0<r_{1}<r_{2}$, there is a Poisson process $X$ with rate 1 , a Poisson process $Y_{1}$ with rate $r_{1}$, and another Poisson process $Y_{2-1}$ with rate $r_{2}-r_{1}$. For any integer $n>0$, let $T(n)$ be the smallest real number such that there are $n+1 X$-points in $J_{T(n)}$. Suppose next that $M_{1}(n)$ is the (random) number of $Y_{1}$-points in $J_{T(n)}$, and $M_{2-1}(n)$ is the (random) number of $Y_{2-1}$-points in $J_{T(n)}$. We refer to the merged $Y_{1}$-points and $Y_{2-1}$-points as $Y_{2}$-points. We define $\Gamma_{n, M_{1}(n)}$ as the domination number generated by the $X$-points and $Y_{1}$-points in $J_{T(n)}$, and $\Gamma_{n, M_{2}(n)}$ as the domination number generated by the $X$-points and $Y_{2}$-points in $J_{T(n)}$. Basically, we have just added $M_{2-1}(n) Y_{2-1}$-points to those $M_{1}(n) Y_{1}$-points to allow us to study the change from $\Gamma_{n, M_{1}(n)}$ to $\Gamma_{n, M_{2}(n)}$. Since adding $Y$-points can never decrease the domination number, we know that $\Gamma_{n, M_{2}(n)}$ is larger than or equal to $\Gamma_{n, M_{1}(n)}$. Recall that $Y_{1}$-points are generated from a Poisson process with rate $r_{1}$, and $Y_{2-1}$-points are generated from a Poisson process with 
rate $r_{2}-r_{1}$, hence $Y_{2}$-points are generated from a Poisson process with rate $r_{2}$. Therefore, by previous results, we have

$$
\lim _{n \rightarrow \infty} \frac{\Gamma_{n, M_{1}(n)}}{\left|J_{T(n)}\right|}=g_{2}\left(r_{1}\right) \text { a.s. } \quad \text { and } \quad \lim _{n \rightarrow \infty} \frac{\Gamma_{n, M_{2}(n)}}{\left|J_{T(n)}\right|}=g_{2}\left(r_{2}\right) \text { a.s. }
$$

Recalling $\Gamma_{n, M_{2}(n)}$ is larger than or equal to $\Gamma_{n, M_{1}(n)}$, we conclude that $g_{2}\left(r_{2}\right) \geq g_{2}\left(r_{1}\right)$.

- $g_{2}(r)$ is continuous: For any $r_{1}, r_{2}>0$ and $\epsilon>0$, we must show that there exists a $\delta=\delta(\epsilon)>0$ such that if $\left|r_{2}-r_{1}\right|<\delta$, then $\left|g_{2}\left(r_{2}\right)-g_{2}\left(r_{1}\right)\right| \leq \epsilon$. Suppose there is a Poisson process $X$ with rate 1 , a Poisson process $Y_{1}$ with rate $r_{1}$, and another Poisson process $Y_{2}$ with rate $r_{2}$. Then for any integer $n>0$, we let $T(n)$ be the smallest real number such that there are $n+1 X$-points in $J_{T(n)}$. Suppose next that $M_{1}(n)$ is the (random) number of $Y_{1}$-points in $J_{T(n)}$, and $M_{2}(n)$ is the (random) number of $Y_{2}$-points in $J_{T(n)}$. Taking into consideration that almost sure convergence implies convergence in probability, we have

$$
\lim _{n \rightarrow \infty} \frac{\Gamma_{n, M_{1}(n)}}{\left|J_{T(n)}\right|}=g_{2}\left(r_{1}\right) \text { in probability and } \lim _{n \rightarrow \infty} \frac{\Gamma_{n, M_{2}(n)}}{\left|J_{T(n)}\right|}=g_{2}\left(r_{2}\right) \text { in probability. }
$$

We will prove the continuity of $g_{2}(r)$ by contradiction. Suppose that $\left|r_{1}-r_{2}\right|<\delta$ and that $\mid g_{2}\left(r_{2}\right)-$ $g_{2}\left(r_{1}\right) \mid>\epsilon$, i.e. $\left|g_{2}\left(r_{2}\right)-g_{2}\left(r_{1}\right)\right|=\epsilon+\alpha$ for some $\alpha>0$. By the definition of convergence in probability, we know that when $n$ is sufficiently large,

$$
P\left(\left|\frac{\Gamma_{n, M_{1}(n)}}{\left|J_{T(n)}\right|}-g_{2}\left(r_{1}\right)\right|<\alpha / 2\right)>1-\delta \text { and } P\left(\left|\frac{\Gamma_{n, M_{2}(n)}}{\left|J_{T(n)}\right|}-g_{2}\left(r_{2}\right)\right|<\alpha / 2\right)>1-\delta .
$$

The events in the above probabilities together with $\left|g_{2}\left(r_{2}\right)-g_{2}\left(r_{1}\right)\right|=\epsilon+\alpha$ imply that

$$
P\left(\left|\frac{\Gamma_{n, M_{1}(n)}}{\left|J_{T(n)}\right|}-\frac{\Gamma_{n, M_{2}(n)}}{\left|J_{T(n)}\right|}\right|>\epsilon\right)>1-2 \delta
$$

On the other hand, applying the same techniques used in the proof of Lemma 4.1, we can prove the following result similar to Inequality (A.8). If $\left|r_{2}-r_{1}\right|<\delta$, then when $n$ is sufficiently large,

$$
P\left(\left|\frac{\Gamma_{n, M_{1}(n)}}{\left|J_{T(n)}\right|}-\frac{\Gamma_{n, M_{2}(n)}}{\left|J_{T(n)}\right|}\right|>\epsilon\right) \leq P\left(\left|M_{2}(n)-M_{1}(n)\right| \geq \delta n\right)+\left(C_{1}^{\prime}+C_{2}^{\prime}\right) \delta
$$

where $C_{1}^{\prime}, C_{2}^{\prime}>0$ are two constants determined by $\epsilon$.

Applying Chernoff's Theorem as before, for any fixed $\delta>0$, the probability $P\left(\left|M_{2}(n)-M_{1}(n)\right| \geq \delta n\right)$ converges to 0 as $n$ goes to $\infty$. Therefore, when $\delta$ is sufficiently small, the inequality above can be further bounded as follows: For some constant $C>0$,

$$
P\left(\left|\frac{\Gamma_{n, M_{1}(n)}}{\left|J_{T(n)}\right|}-\frac{\Gamma_{n, M_{2}(n)}}{\left|J_{T(n)}\right|}\right|>\epsilon\right) \leq C \delta \leq 1-2 \delta,
$$

which contradicts Inequality (A.9).

Acknowledgements. The authors thank the Johns Hopkins University's Acheson J. Duncan Fund for the Advancement of Research in Statistics for supporting this research. J.C. Wierman gratefully acknowledges the financial support and stimulating environment of the Mittag-Leffler Institute of the Swedish Royal Academy of Sciences during his sabbatical visit. 


\section{REFERENCES}

[1] M.A. Akcoglu and U. Krengel, Ergodic theorems for superadditive processes. Journal für die Reine und Angewandte Mathematik 323 (1981) 53-67.

[2] A. Bonato, M. Lozier, D. Mitsche, X. Péréz-Giménez and P. Prałat, The domination number of on-line social networks and random geometric graphs. Theory and Applications of Models of Computation. TAMC 2015, edited by R. Jain, S. Jain and F. Stephan. In Vol. 9076 of Lecture Notes in Computer Science. Springer, Cham (2015).

[3] A. Cannon and L. Cowen, Approximation algorithms for the class cover problem. 6th International Symposium on Artificial Intelligence and Mathematics (2000).

[4] E. Ceyhan and C.E. Priebe, The use of domination number of a random proximity catch digraph for testing spatial patterns of segregation and association. Stat. Prob. Lett. 73 (2005) 37-50.

[5] E. Ceyhan, Extension of one-dimensional proximity regions to higher dimensions. Comp. Geom.-Theor. Appl. 43 (2010) $721-748$.

[6] E. Ceyhan, Spatial clustering tests based on domination number of a new family of random digraphs. Commun. Stat. A-Ther. 40 (2011) 1363-1395.

[7] E. Ceyhan, Comparison of relative density of two random geometric digraph families in testing spatial clustering. TEST $\mathbf{2 3}$ (2014) 100-134.

[8] J.G. DeVinney, The Class Cover Problem and its Applications in Pattern Recognition, Ph.D. dissertation, Johns Hopkins University (2003).

[9] J.G. DeVinney and C.E. Priebe, A new family of proximity graphs: class cover catch digraphs. Disc. Appl. Math. 154 (2006) $1975-1982$.

[10] J.G. DeVinney and J.C. Wierman, A SLLN for a one-dimensional class cover problem. Stat. Prob. Lett. 59 (2002) $425-435$.

[11] J.L. Doob, Stochastic Processes. Chapman \& Hall, London (1953).

[12] C.K. Eveland, D.A. Socolinsky, C.E. Priebe and D.J. Marchette, A hierarchical methodology for one-class problems with skewed priors. J. Classif. 22 (2005) 17-48.

[13] M. Haenggi, The secrecy graph and some of its properties. IEEE International Symposium on Information Theory (ISIT'08). Toronto, Canada (2008) 539-543.

[14] J.M. Hammersley and D.J.A. Welsh, First-passage percolation, subadditive processes, stochastic networks and generalized renewal theory, in Bernoulli-Bayes-Laplace Anniversary Volume, edited by L. LeCam and J. Neyman. Proceedings, International Research Seminar, Statistical Laboratory, University of California, Berkeley. Springer Verlag (1965).

[15] T.W. Haynes, S.T. Hedetniemi and P.J. Slater, Domination in Graphs, Fundamentals. Marcel Dekker, Inc., New York (1998).

[16] J.F.C. Kingman, The ergodic theory of subadditive stochastic processes. J. R. Statist. Soc., Ser. B 30 (1968) 499-510.

[17] J.F.C. Kingman, Subadditive ergodic theory. Ann. Probab. 1 (1973) 883-909.

[18] S.R. Kulkarni, G. Lugosi and S.S. Vankatesh, Learning pattern classification - a survey. IEEE Trans. Info. Theory 44 (1998) 2178-2206.

[19] D.J. Marchette and C.E. Priebe, Characterizing the scale dimension of a high-dimensional classification problem. Pattern Recogn. 36 (2003) 45-60.

[20] A. Manukyan and E. Ceyhan, Classification of imbalanced data with a geometric digraph family. J. Mach. Learn. Res. 17 (2016) $1-40$.

[21] O. Ore, Theory of Graphs. American Mathematical Society, Providence, R.I. (1962)

[22] M.D. Penrose and J.E. Yukich, Weak laws of large numbers in geometric probability. Ann. Appl. Probab. 13 (1993) $277-303$.

[23] C.E. Priebe, D.J. Marchette, J.G. DeVinney and D. Socolinsky, Classification using class cover catch digraphs. J. Classif. 20 (2003) 3-23.

[24] C.E. Priebe, J.L. Solka, D.J. Marchette and B.T. Clark, Class cover catch digraphs for latent class discovery in gene expression monitoring by DNA microarrays. Comp. Stat. Data An. on Visualization 43 (2003) 621-632.

[25] W.T. Rhee, A matching problem and subadditive Euclidean functionals. Ann. Appl. Probab. 3 (1993) $794-801$.

[26] A. Sasireka and A.H. Nandhu Kishore, Applications of dominating set of graph in computer networks. Int. J. Eng. Sci. Res. Technol. 3 (2014) 170-173.

[27] A. Sarkar and M. Haenggi, Percolation in the secrecy graph. Discr. Appl. Math. 161 (2013) 2120-2132.

[28] R.T. Smythe, Multiparameter subadditive processes. Ann. Prob. 4 (1976) 772-782.

[29] R.T. Smythe and J.C. Wierman, First-passage Percolation on the Square Lattice. Vol. 671 of Lect. Notes Math. (1978).

[30] J.M. Steele, Subadditive Euclidean functionals and nonlinear growth in geometric probability. Ann. Probab. 4 (1981) 365-376.

[31] J.C. Wierman and P. Xiang, A general SLLN for a one-dimensional class cover problem. Stat. Prob. Lett. 78 (2008) $1110-1118$.

[32] P. Xiang and J.C. Wierman, A CLT for a one-dimensional class cover problem. Stat. Prob. Lett. 79 (2009) $223-233$.

[33] J.E. Yukich, Limit theorems in discrete stochastic geometry, Stochastic Geometry, Spatial Statistics and Random Fields, edited by E. Spodarev. In Vol. 2068 of Lecture Notes in Mathematics. Springer, Berlin, Heidelberg (2013).

[34] Y. Zhao, L. Kang and M.Y. Sohn, The algorithmic complexity of mixed domination in graphs. Theor. Comput. Sci. 412 (2011) $2387-2392$. 